\title{
Stable cohomotopy groups of compact spaces
}

\author{
by \\ Sławomir Nowak (Warszawa)
}

\begin{abstract}
We show that one can reduce the study of global (in particular cohomological) properties of a compact Hausdorff space $X$ to the study of its stable cohomotopy groups $\pi_{s}^{k}(X)$.

Any cohomology functor on the homotopy category of compact spaces factorizes via the stable shape category ShStab. This is the main reason why the language and technique of stable shape theory can be used to describe and analyze the global structure of compact spaces.

For a given Hausdorff compact space $X$, there exists a metric compact space with the same stable shape iff the stable cohomotopy groups of $X$ are countable. If $\pi_{s}^{n}(X)=0$ for almost all $n>0$ and the integral cohomology groups of $X$ are countable (respectively finitely generated) for all $n$, then the $k$-fold suspension of $X$ has the same stable shape as a finite-dimensional compact metric space (respectively a finite CW complex) for sufficiently large $k$.

There is a duality between compact Hausdorff spaces and CW spectra under which stable cohomotopy groups of $X$ correspond to homotopy groups of the CW spectrum $\mathbb{W}_{X}$ assigned to $X$ and the class of all $X$ with $\mathfrak{C}^{s}(X)=\max \left\{k: \pi_{s}^{k}(X) \neq 0\right\}<\infty$ corresponds to the class of spectra bounded below.

The notion of the cohomological dimension $\mathfrak{H}$-dim $X$ with respect to a generalized cohomology theory $\mathfrak{H}$ is studied. In particular we show that $\pi$-dim $X \geq \mathfrak{H}$-dim $X$ for every $\mathfrak{H}$ and $\boldsymbol{\pi}-\operatorname{dim} X=\infty$ if $\boldsymbol{\pi}$-dim $X>\operatorname{dim}_{\mathbb{Z}} X$, where $\boldsymbol{\pi}$ is the stable cohomotopy theory and $\operatorname{dim}_{\mathbb{Z}} X$ is the integral cohomological dimension. The following question remains open: does $\pi$-dim $X$ coincide with $\operatorname{dim} X$ ?
\end{abstract}

Consider a reduced generalized cohomology theory $\mathfrak{H}^{*}=\left\{\mathbf{h}^{n}\right\}$ defined on the homotopy category $\mathcal{H C W}_{f}$ of finite pointed $\mathrm{CW}$ complexes. It consists of a family of contravariant functors $\mathbf{h}^{n}: \mathcal{H C W} \mathcal{W}_{f} \rightarrow \mathcal{A B}$ together with a family of natural equivalences $\varepsilon^{n}: \mathbf{h}^{n+1} \rightarrow \mathbf{h}^{n} \cdot \mathbf{S}$, where $\mathcal{A B}$ denotes the category of Abelian groups and $\mathbf{S}$ the reduced suspension functor ([Sw, p. 124]).

The Cech cohomology groups $h^{n}(X)$ of a compact Hausdorff space $X$ are equal to the direct limit of the system $\left\{h^{n}(|N(\alpha)|)\right\}$, where $\alpha$ varies over the

2000 Mathematics Subject Classification: Primary 55P25, 55P55, 55N20; Secondary $55 \mathrm{~N} 07$.

Key words and phrases: stable shape, spectrum, weak homotopy class, SpanierWhitehead Duality. 
finite open coverings of $X$. The generalized Čech cohomology groups commute with limits of compact spaces (the continuity property). Cohomology functors are particularly suited for applications in which compact spaces are mapped into locally nice spaces such as CW complexes and ANR's.

Suppose that the reduced cohomology theory $\mathfrak{H}^{*}=\left\{\mathbf{h}^{n}\right\}$ is associated with a CW spectrum $\mathbb{E}=\left\{E_{n}\right\}$ and $X$ is a compact Hausdorff space. The $n$th cohomology group $h^{n}(X)$ of $X$ is isomorphic to the direct limit of the sequence $\left\{\left[X, E_{n}\right] \rightarrow\left[\mathbf{S}(X), E_{n+1}\right] \rightarrow\left[\mathbf{S}^{2}(X), E_{n+2}\right] \rightarrow \ldots\right\}$. In particular the stable cohomotopy groups $\pi_{s}^{n}(X)$ of $X$ are isomorphic to the direct limit $\left.\stackrel{\lim }{\longrightarrow}\left\{\mathbf{S}^{k}(X), S^{n+k}\right]\right\}$.

Shape theory (respectively stable shape theory) is a modification of homotopy theory (respectively stable homotopy theory), specially designed for the study of spaces with complicated local properties. The stable shape category ShStab is a proper framework for studying properties of Čech cohomology functors and relationships between them. It is convenient because each stable shape morphism induces a homomorphism of cohomotopy groups and the morphism induced by a homotopy class depends only on the stable shape morphism which is induced by this class.

In the late eighties it was realized that the Cech $S$-category, introduced thirty years before (and nine years before the advent of shape theory) by E. Lima (see [L, p. 112]), was indeed the stable shape category. The paper $[\mathrm{L}]$ contains results important for shape theory in general. One of them corresponds to the Whitehead theorem in homotopy theory and states that the stable shape morphism between compact metric spaces is an isomorphism of the stable shape category if and only if it induces isomorphisms of all stable cohomotopy groups. This implies that if the stable shape morphism induces isomorphisms of stable cohomotopy groups, then it must induce isomorphisms of cohomology groups for any generalized cohomology theory.

For classical shape theory there are results analogous to the Whitehead Theorem for the homotopy category of CW complexes. Roughly speaking, a shape morphism is an isomorphism if and only if it induces isomorphisms of homotopy progroups (see [Ma-Se, p. 143]). In contrast to the classical case, we must assume that the spaces are finite-dimensional in a certain sense. T. Miyata and J. Segal have shown (see $\left[\mathrm{Mi}_{-} \mathrm{Se}_{1}\right]$ ) that under reasonable assumptions the same theorem holds for the generalized stable shape category whose objects are spaces and spectra (this category contains the stable shape category as a subcategory $\left[\mathrm{Mi}^{\left.-\mathrm{Se}_{1}\right]}\right]$ ). Instead of homotopy progroups they consider stable homotopy progroups. In this case a variant of finitedimensionality is also essential (see [Mi-Se 1 , Example 6.3, p. 163]).

The Lima Theorem covers the case of infinite-dimensional compacta. It generalizes the Co-Whitehead Theorem in stable homotopy theory ([F, p. 136]) and is a suitable version of the Whitehead Theorem for stable shape. 
The present paper contains a generalization of the Lima Theorem to the case of all compact Hausdorff spaces (see also Remark 8). Additionally we find conditions which guarantee that the stable cohomotopy groups can be replaced by the cohomology groups with integer coefficients (see Example 1).

Suppose that $\mathfrak{H}^{*}=\left\{\mathbf{h}^{n}\right\}$ is a reduced generalized cohomology theory and $X \neq \emptyset$ is a compact Hausdorff space. The global generalized cohomological dimension of $X$ with respect to $\mathfrak{H}$ is $-\infty$ if $h^{m}(X)=0$ for every $m$, and otherwise it is $\max \left\{n: h^{n}(X) \neq 0\right\}$. In particular, if $X$ has trivial stable shape (i.e. $\operatorname{ShStab}(X)=\operatorname{ShStab}(\{$ point $\}))$, then the global dimension of $X$ with respect to $\mathfrak{H}$ is $-\infty$.

The global generalized cohomological dimension of $X$ with respect to the stable cohomotopy theory is denoted by $\mathfrak{C}^{s}(X)$. Observe that $\mathfrak{C}^{s}\left(S^{0}\right)=0$ and $\mathfrak{C}^{s}(X)$ is either nonnegative (possibly $\infty$ ) or $-\infty$.

Theorem A (cf. Theorem 3.2.2 and Corollary 7.2.5). Let $X$ and $Y$ be compact Hausdorff spaces. A morphism $\underline{\mathbf{f}} \in \operatorname{ShStab}(X, Y)$ is an isomorphism iff it induces isomorphisms of stable cohomotopy groups in dimensions $\geq 0$. If $\mathfrak{C}^{s}(X), \mathfrak{C}^{s}(Y)<\infty$, then $\underline{\mathbf{f}}$ induces isomorphisms of all stable cohomotopy groups if and only if it induces isomorphisms of all Cech cohomology groups with integer coefficients.

As an application we find that the inclusion $i: A \rightarrow X$ of a closed subset $A$ of a compact Hausdorff space $X$ is a stable shape equivalence if and only if $X / A$ has trivial stable shape.

In ShStab it is often useful and important to be able to replace a compact Hausdorff space by a simpler one (metrizable, finite-dimensional, a finite CW complex).

Theorem B (cf. Theorems 7.1.1, 2.3.4, 7.2.1 and Corollaries 7.2.3, 7.2.4). Let $X$ be a compact Hausdorff space. Then:

(1) $X$ has the stable shape of a compactum (i.e. compact metric space) if and only if the stable cohomotopy groups of $X$ are countable.

(2) If $\mathfrak{C}^{s}(X)<\infty$, then $\mathfrak{C}^{s}(X)=\max \left\{n: H^{n}(X ; \mathbb{Z}) \neq 0\right\}$.

(3) If $\mathfrak{C}^{s}(X)<\infty$, then the stable cohomotopy groups are countable (respectively finitely generated) if and only if the integral cohomology groups are countable (respectively finitely generated).

(4) If $\mathfrak{C}^{s}(X)<\infty$, then the integral cohomology groups of $X$ are finitely generated if and only if there exists a finite $C W$ complex $P$ with the same stable shape as the $k$-fold suspension of $X$ for some $k \geq 0$.

(5) If $\max \left\{0, \mathfrak{C}^{s}(X)\right\}=N<\infty$ and the integral cohomology groups of $X$ are countable then there exists a compact metric space $Y$ with the same stable shape as the $(N+2)$-fold suspension of $X$ such that $\operatorname{dim} Y \leq 2 N+2$. 
There is a curious duality between the class of all compact spaces and CW spectra. The class of all spaces satisfying $\mathfrak{C}^{s}(X)<\infty$ corresponds to the class of spectra that are bounded below.

The main purpose of [L] was to generalize the Spanier-Whitehead Duality to the case of compact subsets of $S^{n}$. E. Lima proved that the complement $S^{n} \backslash X$ is a dual object of $X$ and that the group $\left[S^{n} \backslash Y, S^{n} \backslash X\right]_{w}$ of weak homotopy classes is isomorphic to $\operatorname{ShStab}(X, Y)$. The papers [Ba $]$, [Ba $]$, $[\mathrm{D}-\mathrm{P}],[\mathrm{H}-\mathrm{N}],[\mathrm{H}]$ contain generalizations or strengthenings of Lima's result, but their authors focus attention on the case of finite-dimensional spaces.

The existence of an embedding of the stable shape category ShStab into the weak homotopy category of spectra was shown by J. Segal and T. Miyata in $\left[\mathrm{Mi}_{-} \mathrm{Se}_{2}\right]$. The method of proof suggests (compare [Sw, p. 165 and Remark 2 on p. 331]) that they use a different notion of weak homotopy classes between CW spectra than defined here (see also Remark 4).

In 1999 T. Miyata [Mi] obtained an extension of the Spanier-Whitehead Duality to the generalized stable shape category (see Remark 7) and proved that for every compactum $X$ there exists a dual object, a CW spectrum. In general, the papers [Mi], [Mi-Se 1 ] and $\left[\mathrm{Mi}_{-} \mathrm{Se}_{2}\right]$ contain results which are similar or parallel to those presented here (see Remarks 4 and 7).

In 1998, during the Dubrovnik Conference on Geometric Topology, the author presented another construction of the dual CW spectrum for a metrizable compact space. The next theorem generalizes that result.

Theorem C (cf. Theorems 6.1.1, 6.4.1, Corollaries 6.1.3 and 7.1.3). Let $X$ and $Y$ be compact Hausdorff spaces and let $\mathbb{W}_{X}$ and $\mathbb{W}_{Y}$ be $C W$ substitutes of the function spectra (respectively) $\mathbb{E}(X)$ and $\mathbb{E}(Y)$. Then:

(i) There exists an isomorphism $\mathcal{D}_{\left(\mathfrak{W}_{X}, \mathbb{W}_{Y}\right)}^{X, D_{0}}=\mathcal{D} \operatorname{ShStab}(X, Y) \rightarrow$ $\left\langle\mathbb{W}_{Y}, \mathbb{W}_{X}\right\rangle$ of the Abelian group $\operatorname{ShStab}(X, Y)$ onto the group $\left\langle\mathbb{W}_{Y}, \mathbb{W}_{X}\right\rangle$ of all natural transformations from the associated cohomology theory $\mathfrak{H}_{W_{Y}}$ to $\mathfrak{H}_{\mathbb{W}_{X}}$. Both cohomology theories are defined on the homotopy category $\mathcal{H C W}_{f}$ of finite $C W$ complexes.

(ii) If $\mathbb{W}_{Y}$ is countable or $\mathfrak{C}^{s}(X), \mathfrak{C}^{s}(Y)<\infty$, then $\left\langle\mathbb{W}_{Y}, \mathbb{W}_{X}\right\rangle$ is canonically isomorphic to the group $\left[\mathbb{W}_{Y}, \mathbb{W}_{X}\right]_{w}$ of weak homotopy classes.

(iii) $h_{Y}^{n}(X) \cong h_{X}^{n}(Y)$, where $\mathfrak{H}_{\mathbb{W}_{Y}}=\left\{h_{Y}^{n}\right\}$ and $\mathfrak{H}_{\mathbb{W}_{X}}=\left\{h_{X}^{n}\right\}$.

(iv) If $Y$ is a metrizable space, then $h_{\mathbb{E}}^{n}(X \wedge Y) \cong h_{\mathbb{E} \wedge \mathbb{W}_{Y}}^{n}(X)$, where $\left\{h_{\mathbb{E}}^{n}\right\}$ and $\left\{h_{\mathbb{E} \wedge \mathbb{W}_{Y}}^{n}\right\}$ denote the cohomology theories associated with $\mathbb{E}$ and $\mathbb{E} \wedge \mathbb{W}_{Y}$.

(v) If $Y$ is a movable metrizable space, then $\left\langle\mathbb{W}_{Y}, \mathbb{W}_{X}\right\rangle$ is isomorphic to the group $\left[\mathrm{W}_{Y}, \mathbb{W}_{X}\right]$ of homotopy classes.

(vi) $X$ has the stable shape of a compactum if and only if $\mathbb{W}_{X}$ is isomorphic to a countable spectrum. 
(vii) $\mathbb{W}_{X}$ is isomorphic to a finite $C W$ spectrum iff there exist $k>0$ and a finite $C W$ complex $P$ such that $\mathbf{S}^{k}(X)$ has the same stable shape as $P$.

(viii) $\mathbb{W}_{X}$ is isomorphic to a countable $C W$ spectrum bounded below if and only if there exist $k>0$ and a compactum $Y$ such that $\mathbf{S}^{k}(X)$ has the same stable shape as $Y$.

The Lima Theorem for stable shape corresponds to the Whitehead Theorem for category of spectra under the isomorphism $\mathcal{D}$. In light of this fact it also becomes clear why the finite-dimensionality conditions must be fulfilled in the main results of $\left[\mathrm{Mi}-\mathrm{Se}_{1}\right]$. In [Li] it is proved that there exists a nontrivial CW spectrum with all stable cohomotopy groups vanishing. The existence of such a spectrum corresponds to the existence of the AdamsKahn compactum (see [K], [Ma-Se, p. 153] and [Mi-Se ${ }_{1}$, p. 163]) with trivial (stable) homotopy progroups and nontrivial stable cohomotopy groups.

With all these facts in mind we are naturally facing the problem of finding a characterization of $\mathrm{CW}$ spectra which are isomorphic to the images of the compact spaces under the isomorphism $\mathcal{D}$. It is easy to characterize countable CW spectra having this property in geometric terms (cf. Propositions 5.3.1 and 5.3.2). We also prove (cf. Theorem 5.4.1) that if $\mathbb{W}$ is a countable $\mathrm{CW}$ spectrum bounded below with no cells in dimensions $>0$, then there are $m$ and a finite-dimensional compactum $X$ such that $\mathbf{S}^{m}(\mathbb{W})$ is a $\mathrm{CW}$ substitute for $\mathbb{E}(X)$.

Here is an example of application of the above theorems. If $X$ and $Y$ are continua with $\mathfrak{C}^{s}(X), \mathfrak{C}^{s}(Y)<\infty$ and with one nontrivial reduced cohomology group in dimension $n$, then $\operatorname{ShStab}(X)=\operatorname{ShStab}(Y)$ iff $H^{n}(X ; \mathbb{Z}) \cong$ $H^{n}(Y ; \mathbb{Z})$.

T. Miyata and J. Segal [Mi-Se ${ }_{1}$ have introduced the notion of the stable shape dimension $\operatorname{sd}_{\text {spec }}(X)$ of a compactum $X$. We study the relationships between $\operatorname{sd}_{\text {spec }}(X)$ and $\mathfrak{C}^{s}(X)$.

Let $\mathfrak{H}=\left\{\mathbf{h}^{n}\right\}$ be a reduced generalized cohomology theory. Replacing the ordinary cohomology by $\mathfrak{H}$ in the definition of the cohomological dimension, we get the notion of the generalized cohomological dimension of $X$ with respect to $\mathfrak{H}$ (denoted by $\mathfrak{H}$ - $\operatorname{dim} X$ ). In other words the property of having $\mathfrak{H}$-dim $X \leq n$ is obtained as a localization of the property of having the global cohomological dimension with respect to $\mathfrak{H}$ less than or equal to $n$. It is reasonable to assume that $h^{n}(X)=0$ for sufficiently large $n$ if $\operatorname{dim} X<$ $\infty$ or (at least) that the global cohomological dimension $\mathbf{d} \mathfrak{H}=\max \{n$ : $\left.h^{n}\left(S^{0}\right) \neq 0\right\}$ of the 0 -sphere $S^{0}$ is finite. If $\mathfrak{H}$ does not satisfy this condition then $\mathfrak{H}-\operatorname{dim} \mathbf{I}=\infty$, where $\mathbf{I}$ denotes the closed interval $[0,1]$. Since we may reindex $\mathfrak{H}$ by adding $-\mathbf{d} \mathfrak{H}$ to all indices it suffices to consider cohomology theories with $\mathbf{d} \mathfrak{H}=0$. In this case $\mathfrak{H}$ - $\operatorname{dim} \sigma=n$ for every $n$-dimensional closed simplex $\sigma$. 
It is natural to ask whether there is a cohomology theory $\mathfrak{H}$ with $\mathbf{d} \mathfrak{H}=0$ such that $\mathfrak{H}-\operatorname{dim} X=\infty$ if $\operatorname{dim} X=\infty$. We prove that if $X$ is a compactum and there exists $\mathfrak{H}$ such that $\mathfrak{H}$ - $\operatorname{dim} X=\infty$ then the generalized cohomological dimension of $X$ with respect to stable cohomotopy is also infinite, $\pi$-dim $X=\infty$.

\section{PRELIMINARIES}

1.1. Prerequisites. We shall assume that the reader is familiar with the classical shape theory (see [Bo], [Ma-Se]) for compact Hausdorff spaces, and with basic elements of stable homotopy theory ([ $\left.\mathrm{A}_{1}\right],[\mathrm{Ma}]$ and $\left.[\mathrm{Sw}]\right)$.

We recall that "compactum" means a compact metric space.

1.2. Spectra. All spaces considered are pointed. If $\left(X, x_{0}\right)$ is a space then $\mathbf{S}(X)=X \times[-1,1] / X \times\{-1\} \cup\left\{x_{0}\right\} \times[-1,1] \cup X \times\{1\}$ denotes the reduced suspension of $X$. For $(x, t) \in X \times[-1,1]$ we use $x \wedge t$ to denote the corresponding point of $\mathbf{S}(X)$ under the quotient map. If $(x, t)$ does not belong to $X \times\{-1\} \cup\left\{x_{0}\right\} \times[-1,1] \cup X \times\{1\}$, then we shall also use the notation $(x, t)=x \wedge t$.

The $(n+1)$-sphere is the reduced suspension $\mathbf{S}\left(S^{n}\right)$ of the $n$-sphere, and the 0 -sphere consists of two points: -1 and 1 (the first is the base point of $S^{0}$ ). It is convenient to regard $S^{n}$ as the equator of $S^{n+1}$. The base points of $S^{n}$ and $S^{n+1}$ are the same. We shall also identify $\mathbf{S}(X)$ with the smash product $X \wedge S^{1}$.

The set $\{X, Y\}$ of stable homotopy classes from $X$ to $Y$ is the direct limit of the sequence $\left\{[X, Y] \rightarrow[\mathbf{S}(X), \mathbf{S}(Y)] \rightarrow\left[\mathbf{S}^{2}(X), \mathbf{S}^{2}(Y)\right] \rightarrow \ldots\right\}$. The element represented by a map $f: \mathbf{S}^{k}(X) \rightarrow \mathbf{S}^{k}(Y)$ is denoted by $\{f\}$. The set $\{X, Y\}$ is equipped with the structure of an Abelian group. In particular the group $\pi_{n}^{s}(X)=\left\{S^{n}, X\right\}$ is called the $n$-dimensional stable homotopy group of $X$. Similarly the group $\pi_{s}^{n}(X)=\left\{X, S^{n}\right\}$ is called the $n$-dimensional stable cohomotopy group of $X$.

A spectrum $\mathbb{E}$ is a sequence of spaces $E_{n}$ and maps $\varepsilon_{n}: \mathbf{S}\left(E_{n}\right) \rightarrow E_{n+1}$.

If $\mathbb{F}=\left\{F_{n}, \delta_{n}\right\}$ is a spectrum such that $E_{n} \supset F_{n}$ and $\varepsilon_{n}(x)=\delta_{n}(x)$ for $x \in F_{n}$ and $k=1,2, \ldots$, then we say that $\mathbb{F}$ is a subspectrum of $\mathbb{E}$.

We say $\mathbb{E}$ is a $\mathrm{CW}$ spectrum if $E_{n}$ are $\mathrm{CW}$ complexes and each $\varepsilon_{n}$ is a cellular inclusion. A subspectrum $\mathbb{F} \subset \mathbb{E}$ consists of subcomplexes $F_{n} \subset E_{n}$ such that $\mathbf{S}\left(F_{n}\right) \subset F_{n+1}$.

Let $C_{n}$ be the set of cells in $E_{n}$ other than the base point. Elements of the direct limit of the sequence $\left\{\ldots \stackrel{\text { Suspension }}{\longrightarrow} C_{n} \stackrel{\text { Suspension }}{\longrightarrow} C_{n+1} \stackrel{\text { Suspension }}{\longrightarrow} \ldots\right\}$ are stable cells (or cells). Each cell in $E_{n}$ is a member of exactly one cell of $\mathbb{E}$. If the stable cell $\underline{\boldsymbol{\sigma}}$ is represented by a cell $\sigma$ of $E_{n}$, then $\operatorname{dim} \underline{\boldsymbol{\sigma}}=\operatorname{dim} \sigma-n$. 
A CW spectrum $\mathbb{E}$ is called finite (respectively countable) if it has only finitely (countably) many cells.

A subspectrum $\mathbb{E} \subset \mathbb{E}$ is called cofinal if $\mathbb{E}$ and $\mathbb{E}$ have the same sets of stable cells, i.e. for any cell $e$ of $E_{n}$ there is $m$ such that $\mathbf{S}^{m}(e)$ is a cell of $F_{m+n}$.

Let $\mathbb{E}=\left\{E_{n}\right\}$ be a $C W$ spectrum indexed by $n \in \mathbb{Z}$. The spectrum $\mathbb{E}^{\prime}=\left\{E_{n}^{\prime}\right\}$ with

$$
E_{n}^{\prime}= \begin{cases}E_{n} & \text { for } n \geq 0 \\ \{\text { point }\} & \text { for } n<0,\end{cases}
$$

is cofinal in $\mathbb{E}$. For this reason it does not really make any difference whether we consider spectra indexed by $n \in \mathbb{Z}$ or by $n \in\{0,1,2, \ldots\}$. Describing a new $\mathrm{CW}$ spectrum $\mathbb{E}$ it suffices to define its terms $E_{n}$ for $n \geq 0$ and to set $E_{n}=\{$ point $\}$ for $n<0$.

By $\mathbb{S}=\left\{S^{n}\right\}$ we denote the CW spectrum consisting of all spheres.

1.3. Morphisms of spectra and weak homotopy classes. A function $f$ from a $\mathrm{CW}$ spectrum $\mathbb{E}$ to a spectrum $\mathbb{F}$ is a sequence of maps $f_{n}: E_{n} \rightarrow F_{n}$ such that the following diagram is strictly commutative for each $n$ :

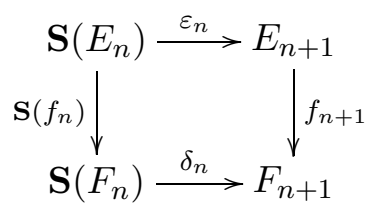

A map from $\mathbb{E}$ to $\mathbb{E}$ is the equivalence class of functions defined on cofinal subspectra of $\mathbb{E}$ with values in $\mathbb{F}$. A morphism from $\mathbb{E}$ to $\mathbb{E}$ is the homotopy class of a map.

In the classical case $([\mathrm{L}]$, cf. $[\mathrm{N}])$ one can also introduce the notion of weakly homotopic maps. Maps $\boldsymbol{\alpha}, \boldsymbol{\beta}: \mathbb{E} \rightarrow \mathbb{E}$ are weakly homotopic if for every finite spectrum $\mathbb{E}_{0}$ and every map $\boldsymbol{\gamma}: \mathbb{E}_{0} \rightarrow \mathbb{E}$ the compositions $\boldsymbol{\alpha} \boldsymbol{\gamma}$ and $\boldsymbol{\beta} \boldsymbol{\gamma}$ are homotopic as maps of spectra. The homotopy class (respectively weak homotopy class) of $\boldsymbol{\alpha}$ is denoted by $[\boldsymbol{\alpha}]$ (respectively $[\boldsymbol{\alpha}]_{w}$ ).

By $[\mathbb{E}, \mathbb{F}]$ (respectively $[\mathbb{E}, \mathbb{F}]_{w}$ ) we denote the set of morphisms from $\mathbb{E}$ to $\mathbb{E}$ (respectively weak homotopy classes of maps from $\mathbb{E}$ to $\mathbb{F}$ ). The sets $[\mathbb{E}, \mathbb{F}]$ and $[\mathbb{E}, \mathbb{F}]_{w}$ are Abelian groups.

For every spectrum $\mathbb{E}$ we can define its suspension $\mathbf{S}(\mathbb{E})=\left\{F_{n}\right\}$, where $F_{n}=E_{n+1}$. This operation has an inverse $\mathbf{S}^{-1}$ defined by $\mathbf{S}^{-1}(\mathbb{E})=\left\{G_{n}\right\}$ and $G_{n}=E_{n-1}$. This allows us to define $\mathbf{S}^{n}$ for every integer $n$.

We define a spectrum $\mathbb{F}$ to be a suspension spectrum if $\varepsilon_{n}$ is a weak homotopy equivalence for sufficiently large $n$. For every space $X$ we denote by $\operatorname{Sus}(X)$ its suspension spectrum (i.e. the spectrum with the $j$-fold suspension 
of $X$ as the $j$ th term). If $X$ and $Y$ are finite CW complexes then

$$
\{X, Y\} \cong[\operatorname{Sus}(X), \operatorname{Sus}(Y)]=[\operatorname{Sus}(X), \operatorname{Sus}(Y)]_{w} .
$$

Let $S \mathcal{S W}$ and $\mathcal{S C W}_{f}$ be the categories of all CW spectra and of all finite CW spectra.

Proposition 1.3.1. Let $\left\{\mathbb{E}_{n}\right\}_{n=1}^{\infty}$ and $\left\{\mathbb{F}_{n}\right\}_{n=1}^{\infty}$ be filtrations of $C W$ spectra $\mathbb{E}$ and $\mathbb{E}$ such that $\mathbb{E}_{n}$ and $\mathbb{F}_{n}$ are finite spectra for every $n$. Then:

(a) There is an exact sequence

$$
0 \rightarrow \lim ^{1}\left[\mathbf{S}\left(\mathbb{E}_{n}\right), \mathbb{F}\right] \rightarrow[\mathbb{E}, \mathbb{F}] \rightarrow \lim _{\longleftarrow}\left[\mathbb{E}_{n}, \mathbb{F}\right] \rightarrow 0 .
$$

(b) $\lim _{\longleftarrow}\left[\mathbb{E}_{n}, \mathbb{E}\right] \cong[\mathbb{E}, \mathbb{E}]_{w} \cong \lim _{n} \lim _{m}\left[\mathbb{E}_{n}, \mathbb{F}_{m}\right]$.

Proof. Part (a) is proved in [Sw, p. 173].

If $\mathbb{E}$ is a suspension $\mathrm{CW}$ spectrum with compact terms, then the set of all morphisms from $\mathbb{E}$ to any $\mathrm{CW}$ spectrum $\mathbb{F}$ can be identified (see [Sw, p. 146]) with the direct limit of the direct sequence $\left\{\ldots \rightarrow\left[E_{n}, F_{n}\right] \rightarrow\right.$ $\left.\left[E_{n+1}, F_{n+1}\right] \rightarrow\left[E_{n+2}, F_{n+2}\right] \rightarrow \ldots\right\}$. This implies (b).

1.4. Categories of inverse and direct systems. Stable shape. If $\mathcal{C}$ is a category, then by Pro- $\mathcal{C}$ we denote the category of inverse systems in $\mathcal{C}$. For abbreviation we write $\left\{X_{\sigma}, p_{\sigma}^{\tau}\right\}$ or $\left\{X_{\sigma}\right\}$ instead of $\left\{X_{\sigma}, p_{\sigma}^{\tau}, \Sigma\right\} \in$ Ob Pro- $\mathcal{C}$. The set of morphisms from $\underline{X}=\left\{X_{\sigma}\right\}$ to $\underline{Y}=\left\{X_{\tau}\right\}$ is defined by

$$
\text { Pro- } \mathcal{C}(\underline{X}, \underline{Y})=\lim _{\leftarrow} \underset{\sigma}{\lim } \mathcal{C}\left(X_{\sigma}, Y_{\tau}\right) \text {. }
$$

Every compact Hausdorff space $X$ is the limit of an inverse system $\underline{\mathbf{X}}=$ $\left\{X_{\sigma}, p_{\sigma}^{\tau}\right\}$ of compact polyhedra with PL bonding maps ([Ma-Se, p. 61]). If $X$ is a compactum then we may assume that $\underline{\mathbf{X}}=\left\{X_{n}, p_{n}^{n+1}\right\}$ is an inverse sequence such that $\operatorname{dim} X \geq \operatorname{dim} X_{n}$.

The system $\underline{\mathbf{X}}$ is mapped into an inverse system $\underline{\widehat{\mathbf{X}}}=\left\{X_{\sigma},\left[p_{\sigma}^{\tau}\right]\right\} \in$ Ob Pro- $\mathcal{H C W}_{f}$ by the homotopy functor, where $\mathcal{H C W}_{f}$ denotes the homotopy category of finite CW complexes. This system is an expansion of $X$ (or is associated with $X)$. The functor Sus assigns to every $\underline{\mathbf{X}} \in \mathrm{Ob}$ Pro- $\mathcal{H C} \mathcal{W}_{f}$ the system $\operatorname{Sus}(\underline{\mathbf{X}})=\left\{\operatorname{Sus}\left(X_{\sigma}\right), \operatorname{Sus}\left(\left[p_{\sigma}^{\tau}\right]\right)\right\} \in \operatorname{Ob} \operatorname{Pro}-\mathcal{S C} \mathcal{W}_{f}$.

Exactly as in the ordinary shape theory (see [Ma-Se, p. 25]) one can define the stable shape category ShStab. The only difference is that we use $\operatorname{Sus}(\underline{\mathbf{X}})$ instead of $\underline{\mathbf{X}} \in \mathrm{Ob}$ Pro- $\mathcal{H C} \mathcal{W}_{f}$ associated with $X$. Roughly speaking, if $\underline{\mathbf{X}}$ and $\underline{\mathbf{Y}}$ are objects of Pro- $\mathcal{H C W}_{f}$ associated respectively with compact Hausdorff spaces $X$ and $Y$ then the morphisms of the stable shape category ShStab from $X$ to $Y$ are represented by the elements of the Abelian group

$$
\begin{aligned}
\operatorname{Pro}-\mathcal{S C W}_{f}(\operatorname{Sus}(\underline{\mathbf{X}}), \operatorname{Sus}(\underline{\mathbf{Y}})) \\
=\lim _{\tau} \lim _{\sigma} \mathcal{S C} \mathcal{W}_{f}\left(\operatorname{Sus}\left(X_{\sigma}\right), \operatorname{Sus}\left(Y_{\tau}\right)\right) \cong \operatorname{ShStab}(X, Y) .
\end{aligned}
$$


We write $\operatorname{ShStab}(X)=\operatorname{ShStab}(Y)$ if $X$ and $Y$ are isomorphic objects in ShStab.

Proposition 1.4.1. If $\operatorname{Sh}\left(\mathbf{S}^{k}(X)\right)=\operatorname{Sh}\left(\mathbf{S}^{k}(Y)\right)$ for a nonnegative integer $k$, then $\operatorname{ShStab}(X)=\operatorname{ShStab}(Y)$.

The description of Pro- $\mathcal{C}$ may be dualized (see [E-H, p. 8]) to yield a category Inj-C of direct systems over $\mathcal{C}$. Morphisms of Inj- $\mathcal{C}$ are defined by the formula

$$
\operatorname{Inj-} \mathcal{C}(\mathbf{X}, \mathbf{Y})=\underset{\leftarrow \sigma}{\lim } \underset{\mu}{\lim } \mathcal{C}\left(X_{\sigma}, Y_{\mu}\right)
$$

where $\mathbf{X}=\left\{X_{\sigma}\right\}$ and $\mathbf{Y}=\left\{Y_{\mu}\right\}$ (in this case we use the same convention as previously, i.e. $\left\{X_{\sigma}, p_{\sigma}^{\tau}, \Sigma\right\} \in \mathrm{Ob}$ Inj-C will be denoted also by $\left\{X_{\sigma}, p_{\sigma}^{\tau}\right\}$ or $\left.\left\{X_{\sigma}\right\}\right)$.

Consider a reduced generalized cohomology theory $\mathfrak{H}^{*}=\left\{\mathbf{h}^{n}\right\}$ defined on the category $\mathcal{S C} \mathcal{W}_{f}$ ([Sw, p. 124]). Together with $\mathfrak{H}^{*}$ we shall also use the corresponding reduced generalized homology theory $\mathfrak{H}_{*}=\left\{\mathbf{h}_{n}\right\}$.

If $\underline{\mathbf{X}}=\left\{\mathbb{X}_{\sigma}, \boldsymbol{\alpha}_{\sigma}^{\tau}\right\} \in$ Ob Pro-SCW $\mathcal{W}_{f}$ and $\underline{\mathcal{A}}=\left\{\mathcal{A}_{\sigma}, \mathbf{f}_{\sigma}^{\tau}\right\} \in \mathrm{ObInj}^{-} \mathcal{S C} \mathcal{W}_{f}$ we set

$$
h^{n}(\underline{\mathbf{X}})=\underline{\lim }\left\{h^{n}\left(\mathbb{X}_{\sigma}\right), h^{n}\left(\boldsymbol{\alpha}_{\sigma}^{\tau}\right)\right\}, \quad h_{n}(\underline{\mathcal{A}})=\underline{\lim }\left\{h_{n}\left(\mathcal{A}_{\sigma}\right), h_{n}\left(\mathbf{f}_{\sigma}^{\tau}\right)\right\} .
$$

In particular if we consider the cohomology and homology theories associated with the sphere spectrum $\mathbb{S}$, then we get the stable cohomotopy groups $\pi^{n}(\underline{\mathbf{X}})$ of the inverse system $\underline{\mathbf{X}} \in$ Ob Pro- $\mathcal{S C W} \mathcal{W}_{f}$ and the stable homotopy groups $\pi_{s}(\underline{\mathcal{A}})$ of $\underline{\mathcal{A}} \in \mathrm{Ob} \operatorname{Inj}-\mathcal{S C} \mathcal{W}_{f}$.

In analogy with the classical case every morphism $\alpha \in \operatorname{Pro} \mathcal{S C W}_{f}(\underline{\mathbf{X}}, \underline{\mathbf{Y}})$ (respectively every $\mathbf{f} \in \operatorname{Inj}-\mathcal{S C} \mathcal{W}_{f}(\underline{\mathcal{A}}, \underline{\mathcal{B}})$ induces a well-defined homomorphism $h^{n}(\boldsymbol{\alpha}): h^{n}(\underline{\mathbf{Y}}) \rightarrow h^{n}(\underline{\mathbf{X}})\left(\right.$ or $\left.h_{n}(\mathbf{f}): h_{n}(\underline{\mathcal{A}}) \rightarrow h_{n}(\underline{\mathcal{A}})\right)$. Moreover, the $n$th generalized cohomology group $h^{n}(\underline{\mathbf{X}})$ is a contravariant functor from Pro- $\mathcal{S C} \mathcal{W}_{f}$ to $\mathcal{A B}$, and the $n$th homology group $h_{n}(\underline{\mathcal{A}})$ is a covariant functor from Inj- $\mathcal{S C} \mathcal{W}_{f}$ to $\mathcal{A B}$.

For every $\mathrm{CW}$ spectrum $\mathbb{E}$ we denote by $\mathfrak{H}_{\mathbb{E}}^{*}$ the cohomology theory associated with $\mathbb{E}$ and defined on $\mathcal{S C W}$. The group of all natural transformations from $\mathfrak{H}_{\mathbb{E}}^{*}$ to $\mathfrak{H}_{\mathbb{F}}^{*}$ is denoted by $\langle\mathbb{E}, \mathbb{E}\rangle$.

Let $\mathbb{E}$ be a CW spectrum and $\underline{\mathbf{F}}=\left\{\mathbb{F}_{\mu}, \mathbf{i}_{\mu}^{\nu}\right\} \in \mathrm{Ob} \operatorname{Inj}-\mathcal{S C W}_{f}$ be a direct system consisting of all finite $\mathrm{CW}$ subspectra $\mathbb{F}_{\mu}$ of $\mathbb{F}$ (ordered by inclusion) and let $\boldsymbol{\alpha} \in \operatorname{Inj}-\mathcal{S C} \mathcal{W}_{f}(\underline{\mathbf{E}}, \underline{\mathbf{F}})$. For every $\mathbb{G} \in \mathrm{Ob} \operatorname{Inj}-\mathcal{S C} \mathcal{W}_{f}$ the morphism $\boldsymbol{\alpha}$ induces a homomorphism $\boldsymbol{\alpha}_{n}^{*}:\left[\mathbb{G}, \mathbf{S}^{n}(\mathbb{E})\right]=h_{\mathbb{E}}^{n}(\mathbb{G}) \rightarrow h_{\mathbb{F}}^{n}(\mathbb{G})=\left[\mathbb{G}, \mathbf{S}^{n}(\mathbb{F})\right]$. It is clear that $\boldsymbol{\alpha}_{n}^{*}$ commutes with the suspension isomorphism, i.e. the collection $\left\{\boldsymbol{\alpha}_{n}^{*}\right\}_{n \in \mathbb{Z}}$ is a natural transformation from $\mathfrak{H}_{\mathbb{E}}^{*}$ to $\mathfrak{H}_{\mathbb{F}}^{*}$ (see [Sw, pp. 115 and 166]).

On the other hand, if $\left\{\boldsymbol{\alpha}_{n}^{*}\right\}_{n \in \mathbb{Z}}: \mathfrak{H}_{\mathbb{E}}^{*} \rightarrow \mathfrak{H}_{\mathbb{E}}^{*}$ is a natural transformation, then for every finite CW subspectrum $\mathbb{E}_{\sigma}$ of $\mathbb{E}$ the transformation $\boldsymbol{\alpha}_{0}^{*}$ assigns to $\mathbb{E}_{\sigma}$ a homomorphism $\boldsymbol{\alpha}_{\mathbb{E}_{\sigma}}: h_{\mathbb{E}}^{0}\left(\mathbb{E}_{\sigma}\right)=\left[\mathbb{E}_{\sigma}, \mathbb{E}\right] \rightarrow\left[\mathbb{E}_{\sigma}, \mathbb{F}\right]=h_{\mathbb{F}}^{0}\left(\mathbb{E}_{\sigma}\right)$. 
Let $\mathbf{f}_{\mathbb{E}_{\sigma}}=\boldsymbol{\alpha}_{\mathbb{E}_{\sigma}}(\mathbf{i}): \mathbb{E}_{\sigma} \rightarrow \mathbb{F}$, where $\mathbf{i}$ denotes the inclusion of $\mathbb{E}_{\sigma}$ into $\mathbb{E}$. The family $\left\{\mathbf{f}_{\mathbb{E}_{\sigma}}\right\}$ represents an element of $\operatorname{Inj}-\mathcal{S C} \mathcal{W}_{f}(\mathbf{E}, \mathbf{F})$, where $\mathbb{E}_{\sigma}$ runs through all finite subspectra of $\mathbb{E}$. This allows us to identify $\langle\mathbb{E}, \mathbb{F}\rangle$ with $\operatorname{Inj}-\mathcal{S C W} \mathcal{W}_{f}(\underline{\mathbf{E}}, \underline{\mathbf{F}})$. Denote by $\langle\boldsymbol{\alpha}\rangle$ the element of $\langle\mathbb{E}, \mathbb{F}\rangle$ represented by $\boldsymbol{\alpha} \in[\mathbb{E}, \mathbb{E}]_{w}$.

Proposition 1.4.2. Let $\mathbb{F}$ be a $C W$ spectrum and consider a system $\underline{\mathbf{E}}=$ $\left\{\mathbb{E}_{\sigma}, \boldsymbol{\alpha}_{\sigma}^{\tau}, \Sigma\right\} \in \mathrm{Ob} \operatorname{Inj}-\mathcal{S C} \mathcal{W}_{f}$. Then Inj-SCW $\mathcal{W}_{f}\left(\left\{\mathbb{E}_{\sigma}, \boldsymbol{\alpha}_{\sigma}^{\tau}, \Sigma\right\}, \underline{\mathbf{F}}\right)$ can be identified with the inverse limit of the system $\left\{\left[\mathbb{E}_{\sigma}, \mathbb{F}\right],\left(\boldsymbol{\alpha}_{\sigma}^{\tau}\right)_{\star}, \Sigma\right\}$, where the function $\left(\boldsymbol{\alpha}_{\sigma}^{\tau}\right)_{\star}:\left[\mathbb{K}_{\tau}, \mathbb{F}\right] \rightarrow\left[\mathbb{K}_{\sigma}, \mathbb{F}\right]$ is induced by $\boldsymbol{\alpha}_{\tau}^{\sigma}$.

Proof. We know ([Sw, p. 146]) that if $\sigma$ is fixed then $\left[\mathbb{E}_{\sigma}, \mathbb{F}\right]=\lim _{\longrightarrow}\left\{\left[\mathbb{E}_{\sigma}, \mathbb{F}_{\mu}\right]\right\}$, where $\underline{\mathbf{F}}=\left\{\mathbb{F}_{\mu}, \mathbf{i}_{\mu}^{\nu}\right\} \in \mathrm{Ob}$ Inj- $\mathcal{S C} \mathcal{W}_{f}$ is the direct system consisting of all finite $\mathrm{CW}$ subspectra $\mathbb{F}_{\mu}$ of $\mathbb{F}$.

Proposition 1.4.3. Let $\mathbb{E}$ and $\mathbb{E}$ be $C W$ spectra. Then:

(a) $[\mathbb{E}, \mathbb{F}]=[\mathbb{E}, \mathbb{E}]_{w}=\langle\mathbb{E}, \mathbb{F}\rangle$ if $\mathbb{E}$ is a finite spectrum.

(b) $\langle\mathbb{E}, \mathbb{F}\rangle=\lim _{1}\left[\mathbb{E}_{\alpha}, \mathbb{F}\right]$, where $\mathbb{E}_{\alpha}$ runs through all finite subspectra of $\mathbb{E}$.

(c) $\langle\mathbb{E}, \mathbb{F}\rangle=[\mathbb{E}, \mathbb{F}]_{w}$ if $\mathbb{E}$ is a countable $C W$ spectrum.

(d) $\langle\mathbb{E}, \mathbb{F}\rangle=[\mathbb{E}, \mathbb{F}]_{w}$ if $\mathbb{E}$ and $\mathbb{F}$ are bounded below.

Proof. Part (b) is a special case of the previous proposition, (c) is a simple consequence of the Homotopy Extension Theorem ([Sw, p. 139]); and $(\mathrm{d})$ is a consequence of Theorem (9.30) of $[\mathrm{Sw}]$.

\section{COMPRESSIBILITY OF MAPS AND SHAPE DIMENSIONS}

2.1. Compressibility of maps. E. Spanier and J. H. C. Whitehead ( $\left[\mathrm{S}-\mathrm{W}_{2}\right.$, p. 92$]$ or $\left[\mathrm{S}-\mathrm{W}_{3}\right.$, p. 359]) studied the problem of compressing $f$ : $(X, A) \rightarrow(Y, B)$ into $Y^{\prime} \supset B$ by a homotopy $\phi:(X \times I, A \times I) \rightarrow(Y, B)$ and described obstructions to compressing $f$ into $Y^{(n-1)} \cup B$ if $f(X) \subset Y^{(n)} \cup B$.

Let us mention the following elementary consequence of their methods (see for instance Theorem (2.1) of $\left[\mathrm{S}-\mathrm{W}_{2}\right]$ ).

Lemma 2.1.1. Let $f:(X, A) \rightarrow(Y, B)$ be a map of pairs of finite $C W$ complexes such that $\operatorname{dim} X \leq n+r-1$ and $Y=Y^{(n-1)} \cup B$ is r-connected, where $2 \leq r \leq n-1$. If $\pi^{n}(f): \pi^{n}(Y, B) \rightarrow \pi^{n}(X, A)$ is a null homomorphism, then there exists $g:(X, A) \rightarrow(Y, B)$ such that $f$ is homotopic to $g$ rel $A$ and $g(X) \subset Y^{(n-1)} \cup B$.

Lemma 2.1.2. Let $f_{i}:\left(\mathbf{S}^{\xi(i)}\left(P_{i}\right), \mathbf{S}^{\xi(i)}\left(Q_{i}\right)\right) \rightarrow\left(\mathbf{S}^{\xi(i)}\left(P_{i-1}\right), \mathbf{S}^{\xi(i)}\left(Q_{i-1}\right)\right)$, where $\xi(i)$ is a natural number and $\left(P_{i}, Q_{i}\right)$ is a pair of connected finite $C W$ complexes for $i=0,1, \ldots, n$. Suppose also that:

(a) $P_{i}^{(r)}=Q_{i}^{(r)}$ for $i=0,1, \ldots, n$.

(b) $\operatorname{dim} P_{0} \leq n+r$. 
(c) $\pi_{s}^{n+r-i+1}\left(\left\{f_{i}\right\}\right): \pi_{s}^{n+r-i+1}\left(P_{i-1} / Q_{i-1}\right) \rightarrow \pi_{s}^{n+r-i+1}\left(P_{i} / Q_{i}\right)$ is a null homomorphism for $i=1, \ldots, n$.

Then for every $m \geq \max \left(\operatorname{dim} P_{0}, \ldots, \operatorname{dim} P_{n}, \xi(1), \ldots, \xi(n)\right)$ there exists a map $g:\left(\mathbf{S}^{m}\left(P_{n}\right), \mathbf{S}^{m}\left(Q_{n}\right)\right) \rightarrow\left(\mathbf{S}^{m}\left(P_{0}\right), \mathbf{S}^{m}\left(Q_{0}\right)\right)$ such that $g\left(\mathbf{S}^{m}\left(P_{n}\right)\right)$ $\subset \mathbf{S}^{m}\left(Q_{0}\right)$ and $g$ is homotopic to the composition $h_{1} \ldots h_{n}$, where $h_{i}=$ $\mathbf{S}^{m-\xi(i)}\left(f_{i}\right)$ for $i=1, \ldots, n$.

Proof. The Suspension Theorem (see $\left[\mathrm{S}_{2}\right.$, p. 458]) and the Excision Theorem for cohomotopy (see [Hu, p. 207]) give

$$
\pi^{l+m}\left(\mathbf{S}^{m}\left(P_{k}\right), \mathbf{S}^{m}\left(Q_{k}\right)\right)=\pi_{s}^{l+m}\left(\mathbf{S}^{m}\left(P_{k}\right) / \mathbf{S}^{m}\left(Q_{k}\right)\right)=\pi_{s}^{l}\left(P_{k} / Q_{k}\right)
$$

for $l=r+1, \ldots, n+r$.

We give $S^{m}$ a CW structure with a single 0-cell and a single $m$-cell.

Since $P_{0}^{(r)}=Q_{0}^{(r)}$ we infer that $\mathbf{S}^{m}\left(P_{0}\right)^{(m+r)}=\left(P_{0} \wedge S^{m}\right)^{(m+r)}=P_{0}^{(r)} \wedge S^{m}$ $=Q_{0}^{(r)} \wedge S^{m}=\left(Q_{0} \wedge S^{m}\right)^{(m+r)}=\mathbf{S}^{m}\left(Q_{0}\right)^{(m+r)}$.

Let $Y_{1}=\mathbf{S}^{m}\left(P_{0}\right)$ and $B=\mathbf{S}^{m}\left(Q_{0}\right)$, and let $Y_{k}$ be the union of the $(n+r+m+1-k)$-skeleton of $Y_{1}$ and $B$ for $k=1, \ldots, n+1$. We infer by (a) that $Y_{n+1}=B$.

Let $j_{k}:\left(Y_{k+1}, B\right) \rightarrow\left(Y_{k}, B\right)$ be the inclusion for $k=1, \ldots, n$.

Let $g_{0}=$ id $:\left(Y_{1}, B\right) \rightarrow\left(Y_{1}, B\right)$. Applying Lemma 2.1.1 for $(Y, B)=$ $\left(Y_{1}, B\right), f=g_{0} h_{1}$ we get a map $g_{1}:\left(\mathbf{S}^{m}\left(P_{1}\right), \mathbf{S}^{m}\left(Q_{1}\right)\right) \rightarrow\left(Y_{2}, B\right)$ such that $j_{1} g_{1}$ is homotopic to $g_{0} h_{1}$ rel $\mathbf{S}^{m}\left(Q_{1}\right)$.

Assume that for $k \geq i \geq 0$ we have a map $g_{i}:\left(\mathbf{S}^{m}\left(P_{i}\right), \mathbf{S}^{m}\left(Q_{i}\right)\right) \rightarrow$ $\left(Y_{i+1}, B\right)$ such that

$$
g_{i} h_{i+1} \simeq j_{i+1} g_{i+1} \operatorname{rel}^{m}\left(Q_{i+1}\right) \quad \text { for } i=1, \ldots, k-1 .
$$

Applying Lemma 2.1.1 for $(Y, B)=\left(Y_{k+1}, B\right), f=g_{k} h_{k+1}$ we obtain a map $g_{k+1}:\left(\mathbf{S}^{m}\left(P_{1}\right), \mathbf{S}^{m}\left(Q_{1}\right)\right) \rightarrow\left(Y_{k+2}, B\right)$ such that (1) is satisfied for $0 \leq i \leq k$. This yields the following diagram:

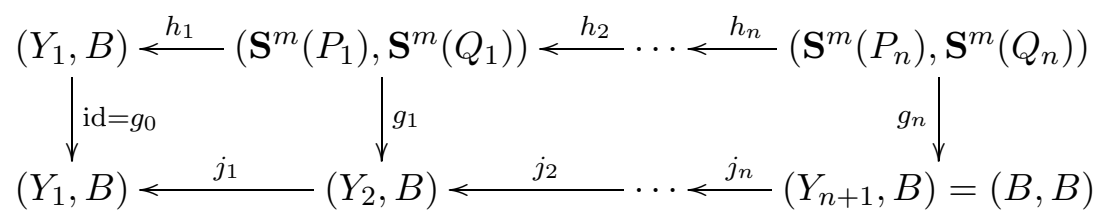

It is clear that $g=j_{1} j_{2} \ldots j_{n} g_{n}$ satisfies the required conditions.

2.2. Shape dimensions of compact spaces. As in the classical shape theory (see [Ma-Se, pp. 106 and pp. 130]) one can introduce the notion of the $n$th stable homotopy pro-group Pro- $\pi_{n}^{s}(X)$ of a compact Hausdorff space $X$, defined to be the pro-group Pro- $\pi_{n}^{s}(\underline{\mathbf{X}})=\left\{\pi_{n}^{s}\left(X_{\sigma}\right), \pi_{n}^{s}\left(p_{\sigma}^{\tau}\right)\right\}$, where $\underline{\mathbf{X}}=\left\{X_{\sigma}, p_{\sigma}^{\tau}\right\}$ is an inverse system of finite CW complexes such that $X$ is homeomorphic to the inverse limit of $\underline{\mathbf{X}}$. More precisely, Pro- $\pi_{n}^{s}(X)$ is 
actually a class of naturally isomorphic pro-groups (see [Ma-Se, p. 121]) and for simplicity we identify Pro- $\pi_{n}^{s}(X)$ with Pro- $\pi_{n}^{s}(\underline{\mathbf{X}})$.

The stable connectivity of $X$ is defined as $\mathfrak{C}_{s}(X)=\min \left\{n: \operatorname{Pro}-\pi_{n}^{s}(X)\right.$ $\neq 0\}-1$. It is an invariant of stable shape. We know that $\mathfrak{C}^{s}(A)=\mathfrak{C}_{s}(A)$ $=\infty$, where $A$ denotes the Adams-Kahn continuum (see $\left[\mathrm{A}_{2}\right],[\mathrm{K}]$ and [Ma-Se, p. 153]). We also have $\mathfrak{C}^{s}(X)=-\infty, \mathfrak{C}_{s}(X)=\infty$ for every $X$ with trivial stable shape.

The notion of the stable shape dimension was introduced by T. Miyata and J. Segal in [Mi-Se 1 ] (see [Mi-Se 1 , p. 159]). Suppose that $\underline{X}=\left\{X_{\sigma}, p_{\sigma}^{\tau}, \Sigma\right\}$ is an inverse system in the homotopy category of finite CW complexes associated with a compact Hausdorff space $X$ and $k, n \in \mathbb{Z}$, where $k \leq n$. The stable shape dimension, $\operatorname{sd}_{\text {spec }}(X)$, satisfies $k \leq \operatorname{sd}_{\text {spec }}(X) \leq n$ if for every $\sigma \in \Sigma$ there exist $\tau \geq \sigma, m$ and maps $g: \mathbf{S}^{m}\left(X_{\tau}\right) \rightarrow P, h: P \rightarrow \mathbf{S}^{m}\left(X_{\sigma}\right)$ such that the homotopy class of $h g$ equals $\mathbf{S}^{m}\left(p_{\tau}^{\sigma}\right)$, where $P$ is a $\mathrm{CW}$ complex with $\operatorname{dim} P \leq n+m$ and with no cells of dimension $\leq k+m$ except for a single 0 -cell $*$.

TheOREM 2.2.1. Let $X$ be a compact Hausdorff space with $\mathfrak{C}^{s}(X)<\infty$. If $\mathfrak{C}_{s}(X) \geq \mathfrak{C}^{s}(X)$, then $\operatorname{ShStab}(X)=\operatorname{ShStab}(\{$ point $\})$. If $\operatorname{ShStab}(X) \neq$ $\operatorname{ShStab}(\{$ point $\})$, then $r \leq \operatorname{sd}_{\text {spec }}(X) \leq \mathfrak{C}^{s}(X)$ for every $r \leq \mathfrak{C}_{s}(X)$. If $k \leq \operatorname{sd}_{\text {spec }}(X) \leq n$, then $\pi_{s}^{m}(X)=0$ for every $m>n$.

Proof. Suppose that $\left\{X_{\sigma}, p_{\sigma}^{\tau}, \Sigma\right\}$ is an inverse system in the homotopy category of finite CW complexes associated with $X$. For a given index $\sigma$ we can find $\sigma^{\prime} \geq \sigma$ such that $p_{\sigma}^{\sigma^{\prime}}$ induces a trivial homomorphism of stable homotopy groups in dimensions $\leq r$ (compare [Ma-Se, p. 137, Lemma 2]).

We first assume that $\operatorname{dim} X_{\sigma^{\prime}}>\mathfrak{C}^{\mathfrak{s}}(X)$.

Since the stable cohomotopy groups of finite CW complexes are finitely generated we infer that there exists a finite sequence of indices $\sigma^{\prime}=\tau_{0}<$ $\tau_{1}<\ldots<\tau_{l}=\tau$ such that $p_{\tau_{i}}^{\tau_{i+1}}$ induces a trivial homomorphism of stable cohomotopy groups $\pi_{s}^{j-i}\left(X_{\tau_{i}}\right) \rightarrow \pi_{s}^{j-i}\left(X_{\tau_{i+1}}\right)$ for every $i=0, \ldots, l=j-$ $\mathfrak{C}^{s}(X)-1$, where $j=\operatorname{dim} X_{\tau_{0}}>\mathfrak{C}^{s}(X)$. By Lemma 2.1.2 there exist $m$ and $g: \mathbf{S}^{m}\left(X_{\tau}\right) \rightarrow Q$ such that the homotopy class of $i g$ is equal to $p_{\sigma}^{\tau}$, where $Q$ is the $\left(m+\mathfrak{C}^{s}(X)\right)$-skeleton of $\mathbf{S}^{m}\left(X_{\sigma^{\prime}}\right)$ and $i$ denotes the inclusion $Q \subset \mathbf{S}^{m}\left(X_{\sigma^{\prime}}\right)$.

Since $\mathbf{S}^{m}\left(p_{\sigma}^{\sigma^{\prime}}\right)$ induces a trivial homomorphism of homotopy groups in dimensions $\leq m+r$ (for sufficiently large $m$ ), we can extend $i: Q \rightarrow \mathbf{S}^{m}\left(X_{\sigma^{\prime}}\right)$ over the union of $Q$ and the cone over the $(m+r)$-skeleton of $Q$. This CW complex has the same homotopy type as $P=P / P^{(m+r)}$.

If $\operatorname{dim} X_{\sigma^{\prime}} \leq \mathfrak{C}^{s}(X)$ the proof is similar, but simpler. Indeed in this case the space $P=\mathbf{S}^{m}\left(X_{\sigma^{\prime}}\right) /\left(\mathbf{S}^{m}\left(X_{\sigma^{\prime}}\right)\right)^{(m+r)}$ satisfies the requirements of the definition of $\operatorname{sd}_{\text {spec }}(X)$.

In both cases if $r \geq \mathfrak{C}^{s}(X)$, then $P$ is contractible. 
Hence $r \leq \operatorname{sd}_{\text {spec }}(X) \leq \mathfrak{C}^{s}(X)$ when $\operatorname{ShStab}(X) \neq \operatorname{ShStab}(\{$ point $\})$, and $\operatorname{ShStab}(X)=\operatorname{ShStab}(\{$ point $\})$ when $\mathfrak{C}_{s}(X) \geq \mathfrak{C}^{\mathfrak{s}}(X)$.

The third assertion is a consequence of the fact that $\pi_{s}^{n}(X)$ is the direct limit of $\left\{\pi_{s}^{n}\left(X_{\sigma}\right)\right\}$.

\subsection{Compacta $X$ with $\mathfrak{C}^{s}(X)<\infty$}

LEMMA 2.3.1. Let $X$ be a compactum with $\mathfrak{C}^{s}(X)<\infty$ and set $N=$ $\max \left\{\mathfrak{C}^{s}(X), 0\right\}$. Then there exists a compactum $Y$ such that $\operatorname{dim} Y \leq 2 N+2$ and $\operatorname{ShStab}(Y)=\operatorname{ShStab}\left(\mathbf{S}^{N+2}(X)\right)$.

Proof. We may assume that $X$ is homeomorphic to the inverse limit of a sequence $\underline{\mathbf{X}}=\left\{X_{k}, p_{k}^{k+1}\right\}$ of finite CW complexes such that $\pi_{s}^{n}\left(p_{k}^{k+1}\right)$ : $\pi_{s}^{n}\left(X_{k}\right) \rightarrow \pi_{s}^{n}\left(X_{k+1}\right)$ is a null homomorphism for $n>N$.

Let $P_{i}=\mathbf{S}^{2}\left(X_{k+i}\right), Q_{i}=\mathbf{S}^{2}\left(X_{k+i}^{(N)}\right)$ and $f_{i+1}=\mathbf{S}^{2}\left(p_{k+i}^{k+i+1}\right):\left(P_{i}, Q_{i}\right) \rightarrow$ $\left(P_{i-1}, Q_{i-1}\right)$ for $i=0, \ldots, l-k$. The exactness of the sequence $\ldots \rightarrow$ $\pi_{s}^{q-1}\left(Q_{i}\right) \rightarrow \pi_{s}^{q}\left(P_{i} / Q_{i}\right) \rightarrow \pi_{s}^{q}\left(P_{i}\right) \rightarrow \ldots$ implies that $\pi_{s}^{q}\left(f_{i}\right): \pi_{s}^{q}\left(P_{i} / Q_{i}\right) \rightarrow$ $\pi_{s}^{q}\left(P_{i+1} / Q_{i+1}\right)$ is a null homomorphism for $q>N+2$. This means that $P_{i}, Q_{i}$ and $f_{i}$ satisfy the conditions (a)-(c) of Lemma 2.1.2. Hence for every $k$ there exists

$$
g: \mathbf{S}^{m}\left(X_{l}\right) \rightarrow \mathbf{S}^{m}\left(X_{k}^{(N)}\right) \quad \text { such that } j_{k} g \simeq \mathbf{S}^{m}\left(p_{k}^{l}\right)
$$

where $j_{k}: \mathbf{S}^{m}\left(X_{k}^{(N)}\right) \rightarrow \mathbf{S}^{m}\left(X_{k}\right)$ is the inclusion, $m=\operatorname{dim} X_{l}+2$ and $l=\max \left\{k+1, k+N-\operatorname{dim} X_{k}\right\}$.

Using (2) and replacing $\underline{\mathbf{X}}$ by a suitable subsequence we can require that for every $k$ there exists a cellular map $g_{k}: \mathbf{S}^{m}\left(X_{k+1}\right) \rightarrow \mathbf{S}^{m}\left(X_{k}^{(N)}\right)$ such that $g_{k}^{k+1}=j_{k} g_{k} \simeq \mathbf{S}^{m}\left(p_{k}^{k+1}\right)$, where $m=\operatorname{dim} X_{k+1}+2$.

By the Freudenthal Suspension Theorem there exists $q_{k}^{k+1}: \mathbf{S}^{N+2}\left(X_{k+1}^{(N)}\right)$ $\rightarrow \mathbf{S}^{N+2}\left(X_{k}^{(N)}\right)$ such that $q_{k}^{k+1} \simeq \mathbf{S}^{m-N-2}\left(\left.g_{k}^{k+1}\right|_{X_{k+1}^{(N)}}\right)$. Let $Y_{k}=$ $\mathbf{S}^{N+2}\left(X_{k}^{(N)}\right)$ and $Y=\lim _{\longleftarrow}\left\{Y_{k}, q_{k}^{k+1}\right\}$.

We denote respectively by $\mathbf{g}_{k}^{k+1}: \operatorname{Sus}\left(X_{k+1}\right) \rightarrow \operatorname{Sus}\left(X_{k}\right)$, $\mathbf{q}_{k}^{k+1}: \operatorname{Sus}\left(X_{k+1}\right) \rightarrow \operatorname{Sus}\left(X_{k}\right), \mathbf{j}_{k}: \operatorname{Sus}\left(X_{k}^{(N)}\right) \rightarrow \operatorname{Sus}\left(X_{k}\right)$ and $\mathbf{g}_{k}: \operatorname{Sus}\left(X_{k+1}\right) \rightarrow \operatorname{Sus}\left(X_{k}^{(N)}\right)$ the morphisms of spectra represented by the $\operatorname{maps} g_{k}^{k+1}: \mathbf{S}^{m}\left(X_{k+1}\right) \rightarrow \mathbf{S}^{m}\left(X_{k}\right), q_{k}^{k+1}: \mathbf{S}^{N+2}\left(X_{k+1}^{(N)}\right) \rightarrow \mathbf{S}^{N+2}\left(X_{k}^{(N)}\right)$, $j_{k}: \mathbf{S}^{m}\left(X_{k}^{(N)}\right) \rightarrow \mathbf{S}^{m}\left(X_{k}\right)$ and $g_{k}: \mathbf{S}^{m}\left(X_{k+1}\right) \rightarrow \mathbf{S}^{m}\left(X_{k}^{(N)}\right)$. We have

$$
\mathbf{g}_{k}^{k+1}=\mathbf{j}_{k} \mathbf{g}_{k}, \quad \mathbf{q}_{k}^{k+1}=\mathbf{g}_{k} \mathbf{j}_{k+1} \quad \text { for every } k .
$$

It is evident that $\operatorname{Sus}(\underline{\mathbf{X}})=\left\{\operatorname{Sus}\left(X_{k}\right), \operatorname{Sus}\left(p_{k}^{k+1}\right)\right\}$ and $\left\{\operatorname{Sus}\left(X_{k}\right), \mathbf{g}_{k}^{k+1}\right\}$ are isomorphic objects of Pro- $\mathcal{S C} \mathcal{W}_{f}$.

By (3) we see that $\underline{\boldsymbol{\tau}}=\left\{\mathbf{j}_{k}\right\}:\left\{\operatorname{Sus}\left(X_{k}^{(N)}\right), \mathbf{q}_{k}^{k+1}\right\} \rightarrow\left\{\operatorname{Sus}\left(X_{k}\right), \mathbf{g}_{k}^{k+1}\right\}$ is an isomorphism in the category Pro- $\mathcal{S C W} \mathcal{W}_{f}$ with inverse $\underline{\boldsymbol{v}}=\left\{\mathbf{g}_{k}\right\}$ : 
$\left\{\operatorname{Sus}\left(X_{k}\right), \mathbf{g}_{k}^{k+1}\right\} \rightarrow\left\{\operatorname{Sus}\left(X_{k}^{(N)}\right), \mathbf{q}_{k}^{k+1}\right\}$. Hence $\mathbf{S}^{N+2}(\underline{\mathbf{X}})=\left\{\mathbf{S}^{N+2}\left(\operatorname{Sus}\left(X_{k}\right)\right)\right.$, $\left.\mathbf{S}^{N+2}\left(\mathbf{g}_{k}^{k+1}\right)\right\}$ and $\left\{\operatorname{Sus}\left(Y_{k}\right), \operatorname{Sus}\left(q_{k}^{k+1}\right)\right\}=\left\{\mathbf{S}^{N+2}\left(\operatorname{Sus}\left(X_{k}^{(N)}\right)\right), \mathbf{S}^{N+2}\left(\mathbf{q}_{k}^{k+1}\right)\right\}$ are isomorphic and $\mathbf{S}^{N+2}(X)$ has the same stable shape as $Y$.

Lemma 2.3.2. Let $X$ be a shape $r$-connected continuum with $\mathfrak{C}_{s}(X) \geq$ $2 r \geq 2$. Then $X$ is shape $2 r$-connected.

Proof. We may assume that $X$ is homeomorphic to the inverse limit of a sequence $\left\{X_{k}, p_{k}^{k+1}\right\}$ such that $X_{k}$ is an $r$-connected CW complex and $\pi_{l}^{s}\left(p_{k}^{k+1}\right): \pi_{l}^{s}\left(X_{k+1}\right) \rightarrow \pi_{l}^{s}\left(X_{k}\right)$ is a null homomorphism for every $k$ and $l \leq 2 r$. This means that

$$
\begin{aligned}
\pi_{l+m}\left(p_{k}^{k+1}\right): \pi_{l+m}\left(X_{k+1}\right) & =\left[S^{l+m}, \mathbf{S}^{m}\left(X_{k+1}\right)\right] \rightarrow\left[S^{l+m}, \mathbf{S}^{m}\left(X_{k}\right)\right] \\
& =\pi_{l+m}\left(X_{k}\right)
\end{aligned}
$$

is a null homomorphism for sufficiently large $m$ and $l \leq 2 r$. The Freudenthal Suspension Theorem implies that $\left[S^{l+m}, \mathbf{S}^{m}\left(X_{k}\right)\right] \cong\left[S^{l}, X_{k}\right]$ for every $k$.

Lemma 2.3.3. Let $X$ be a shape $r$-connected continuum with $\operatorname{dim} X=$ $m<2 r$, where $1<r \leq m$. Then there exists a continuum $Y$ such that $\mathbf{S}^{2 r-m}(Y)$ is shape equivalent to $X$ and $\operatorname{dim} Y \leq 2 m-2 r$.

Proof. We may assume that $X$ is homeomorphic to the inverse limit of a sequence $\left\{X_{k}, p_{k}^{k+1}\right\}$, where each $X_{k}$ is an $r$-connected CW complex with $\operatorname{dim} X_{k} \leq m$. It is known (see for example [S $\mathrm{S}_{2}$, p. 461, Exercise D1]) that for every $r$-connected $\mathrm{CW}$ complex $Q$ with $\operatorname{dim} Q<2 r$ there exists a $\mathrm{CW}$ complex $P$ such that $\mathbf{S}(P)$ is homotopy equivalent to $Q$.

The above fact and the Freudenthal Suspension Theorem imply that for every $k$ there exist a CW complex $Y_{k}$ and a map $q_{k}^{k+1}: Y_{k+1} \rightarrow Y_{k}$ such that the diagram

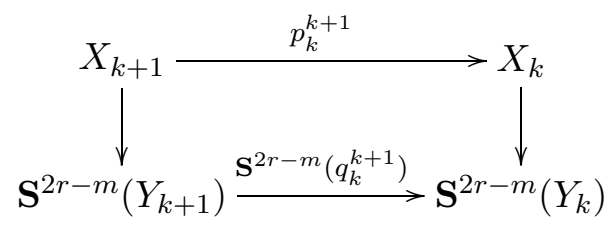

commutes up to homotopy. The vertical arrows are homotopy equivalences. We can assume (see [W, Proposition 4.1, p. 68]) that $\operatorname{dim} Y_{k} \leq 2 m-2 r$ for every $k$. The inverse limit $Y$ of the sequence $\left\{Y_{k}, q_{k}^{k+1}\right\}$ satisfies the required conditions.

Theorem 2.3.4. Let $X$ be a compactum satisfying $\operatorname{ShStab}(X) \neq$ $\operatorname{ShStab}(\{$ point $\}), \mathfrak{C}^{s}(X)=N<\infty$ and $\mathfrak{C}_{s}(X)=r$. Then there exists a shape $(N-r)$-connected continuum $Y$ satisfying the following conditions:

(1) $\operatorname{ShStab}\left(\mathbf{S}^{m_{1}}(X)\right)=\operatorname{ShStab}\left(\mathbf{S}^{m_{2}}(Y)\right)$, where $m_{1}=\max \{0, N-2 r\}$ and $m_{2}=-\min \{0, N-2 r\}$.

(2) $\operatorname{dim} Y \leq 2(N-r)$. 
Proof. By Lemma 2.3.1 there is $X^{\prime}$ such that $\operatorname{dim} X^{\prime}=2 N+2=$ $\mathfrak{C}^{s}\left(X^{\prime}\right)$ and $\operatorname{ShStab}\left(X^{\prime}\right)=\operatorname{ShStab}\left(\mathbf{S}^{N+2}(X)\right)$. We see at once that $\mathfrak{C}_{s}\left(X^{\prime}\right)=$ $N+r+2$ and $\mathfrak{C}^{s}\left(X^{\prime}\right)-\mathfrak{C}_{s}\left(X^{\prime}\right)=N-r$. By Lemma 2.3.2 we conclude that $Z=\mathbf{S}^{N+r+2}\left(X^{\prime}\right)$ is a shape $2(N+r+2)$-connected continuum with $\operatorname{dim} Z=\mathfrak{C}^{s}(Z)=3 N+r+4$. By Lemma 2.3.3 there is $Y$ such that $\mathbf{S}^{N+3 r+4}(Y)$ and $Z$ have the same shape and $\operatorname{dim} Y=\mathfrak{C}^{s}(Y)=2(N-r)$. We have $\operatorname{ShStab}\left(\mathbf{S}^{2 N+r+4}(X)\right)=\operatorname{ShStab}\left(\mathbf{S}^{N+3 r+4}(Y)\right)$. Finally, Proposition 1.4.1 implies that $Y$ satisfies the required conditions.

Corollary 2.3.5. Let $X$ be a compactum with $\mathfrak{C}^{s}(X)<r$. Then the group $\{X, P\}$ of all stable homotopy classes is trivial for every $r$-connected $C W$ complex $P$.

\section{THE WHITEHEAD THEOREMS}

3.1. Formal duality between Pro- $S \mathcal{C} \mathcal{W}_{f}$ and Inj-SCW $\mathcal{W}_{f}$. For each $\mathbb{E} \in \mathrm{ObSCW}_{f}$ there is a finite spectrum $\mathbf{D}(\mathbb{E})$ (the Spanier-Whitehead dual of $\mathbb{E}$ ) and for every pair of $\mathbb{E}, \mathbb{E} \in \mathrm{Ob} \mathcal{S C W}_{f}$ there is an isomorphism $\mathbf{D}_{(\mathbb{E}, \mathbb{F})}=\mathbf{D}:[\mathbb{E}, \mathbb{F}] \rightarrow[\mathbf{D}(\mathbb{E}), \mathbf{D}(\mathbb{E})]\left(\right.$ see $\left[\mathrm{A}_{1}\right.$, p. 190], $[\mathrm{Ma}$, p. 19] or $[\mathrm{Sw}$, p. 321]). This defines a unique, up to natural equivalences, contravariant functor $\mathbf{D}: \mathcal{S C W}_{f} \rightarrow \mathcal{S C W}_{f}$. The following conditions are satisfied:

(1) $\mathbf{D}^{2}$ is naturally equivalent to the identity functor $\mathbf{I}$.

(2) $\mathbf{D}(\mathbf{S}(\mathbb{E}))=\mathbf{S}^{-1}(\mathbf{D}(\mathbb{E}))$.

(3) There is a natural isomorphism $[\mathbb{E} \wedge \mathbb{F}, \mathbb{G}] \cong[\mathbb{E}, \mathbf{D}(\mathbb{F}) \wedge \mathbb{G}]$.

(4) For every generalized homology theory $\mathfrak{H}_{*}$ there exists a natural isomorphism between $h_{k}(\mathbb{E})$ and $h^{-k}(\mathbf{D}(\mathbb{E}))$.

REMARK 1. It is also possible to describe the Spanier-Whitehead dual in a very concrete fashion for the case when $\mathbb{E}=\operatorname{Sus}(X)$ and $X$ is a polyhedron.

It is known that it is possible to embed the space $X$ in the $(n+1)$-sphere for sufficiently large $n$. Suppose that $X_{1} \subset X_{2}$ are subpolyhedra of $S^{n+1}$ and a subpolyhedron $Y_{i} \subset S^{n+1} \backslash X_{i}$ of $S^{n+1}$ is a deformation retract of $S^{n+1} \backslash X_{i}$ such that $Y_{2} \subset Y_{1}$ for $i=1,2$. We may also assume that $X_{i}$ is a deformation retract of $S^{n+1} \backslash Y_{i}$ for $i=1,2$ (possible for larger $n$ ). Then (up to natural equivalences of functors) $\mathbf{D}\left(\operatorname{Sus}\left(X_{i}\right)\right)=\mathbf{S}^{-n}\left(\operatorname{Sus}\left(Y_{i}\right)\right)$, $\mathbf{D}\left(\operatorname{Sus}\left(Y_{i}\right)\right)=\mathbf{S}^{-n}\left(\operatorname{Sus}\left(X_{i}\right)\right), \mathbf{D}(\mathbf{i})=\mathbf{j}$ and $\mathbf{D}(\mathbf{j})=\mathbf{i}$ for $i=1,2$, where $\mathbf{i}$ and $\mathbf{j}$ denote respectively the morphisms of spectra induced by the inclusions $i: X_{1} \rightarrow X_{2}$ and $j: Y_{2} \rightarrow Y_{1}$.

This formula can be generalized to the case when $X_{i}$ are finite CW complexes.

Definition 1. For every $\underline{\mathbf{X}}=\left\{\mathbf{X}_{\sigma}, \boldsymbol{\alpha}_{\sigma}^{\tau}, \Sigma\right\} \in$ Ob Pro- $\mathcal{S C} \mathcal{W}_{f}$ (respectively for every $\left.\underline{\mathcal{A}}=\left\{\mathcal{A}_{\sigma}, \mathbf{f}_{\sigma}^{\tau}, \Upsilon\right\} \in \mathrm{ObInj}-\mathcal{S C} \mathcal{W}_{f}\right)$ the object $\mathbf{D}_{1}(\underline{\mathbf{X}})=$ 
$\left\{\mathbf{D}\left(\mathbb{K}_{\sigma}\right), \mathbf{D}\left(\boldsymbol{\alpha}_{\sigma}^{\tau}\right), \Sigma\right\} \in$ Ob Inj-SCSW $\mathcal{W}_{f}$ (respectively $\mathbf{D}_{2}(\underline{\mathcal{A}})=\left\{\mathbf{D}\left(\mathcal{A}_{\sigma}\right)\right.$, $\left.\mathbf{D}\left(\mathbf{f}_{\sigma}^{\tau}\right), \Upsilon\right\} \in$ Ob Pro- $\mathcal{S C} \mathcal{W}_{f}$ is called the Spanier-Whitehead dual for $\underline{\mathbf{X}}$ (respectively for $\underline{\mathcal{A}})$.

We also have duality isomorphisms

$$
\begin{aligned}
& \mathbf{D}_{1}: \operatorname{Pro}_{\mathcal{S C W}} \mathcal{S C W}_{f}(\underline{\mathbf{X}}, \underline{\mathbf{Y}}) \rightarrow \operatorname{Inj}-\mathcal{S C W} \mathcal{W}_{f}(\mathbf{D}(\underline{\mathbf{Y}}), \mathbf{D}(\underline{\mathbf{X}})), \\
& \mathbf{D}_{2}: \operatorname{Inj}_{\mathcal{S C}} \mathcal{S C} \mathcal{W}_{f}(\underline{\mathcal{A}}, \underline{\mathcal{B}}) \rightarrow \operatorname{Pro}-\mathcal{S C} \mathcal{W}_{f}(\mathbf{D}(\underline{\mathcal{B}}), \mathbf{D}(\underline{\mathcal{A}}))
\end{aligned}
$$

induced by $\mathbf{D}$.

Next, this also yields contravariant functors $\mathbf{D}_{1}:$ Pro- $S \mathcal{C} \mathcal{W}_{f} \rightarrow \operatorname{Inj}-\mathcal{S C} \mathcal{W}_{f}$ and $\mathbf{D}_{2}: \operatorname{Inj}-\mathcal{S C W} \mathcal{W}_{f} \rightarrow$ Pro-SCW $\mathcal{W}_{f}$.

Theorem 3.1.1. The compositions $\mathbf{D}_{2} \mathbf{D}_{1}:$ Pro- $\mathcal{S C} \mathcal{W}_{f} \rightarrow$ Pro- $_{\mathcal{S C}} \mathcal{W}_{f}$ and $\mathbf{D}_{1} \mathbf{D}_{2}:$ Inj-SCW $\mathcal{W}_{f} \rightarrow$ Inj-SCW $\mathcal{W}_{f}$ are naturally equivalent to the identity functors.

\subsection{The Whitehead theorems for Pro- $S \mathcal{S C} \mathcal{W}_{f}$ and stable shape}

Theorem 3.2.1. A morphism $\underline{\mathbf{f}} \in$ Pro- $\mathcal{S C W} \mathcal{W}_{f}(\underline{\mathbf{X}}, \underline{\mathbf{Y}})$ is an isomorphism iff it induces isomorphisms of all stable cohomotopy groups.

Proof. We may assume that $\underline{\mathbf{X}}=\left\{\boldsymbol{X}_{\sigma}, \boldsymbol{\alpha}_{\sigma}^{\tau}, \Sigma\right\}$ and $\underline{\mathbf{Y}}=\left\{\mathbb{Y}_{\sigma}, \boldsymbol{\beta}_{\sigma}^{\tau}, \Sigma\right\}$ are inverse systems indexed over the same cofinite directed set $\Sigma, \underline{\mathbf{f}}=\left\{\mathbf{f}_{\sigma}\right\}$ is a level morphism and $\mathbb{X}_{\sigma}$ and $\mathbb{Y}_{\sigma}$ are finite suspension spectra ([Ma-Se, p. 12]). Then for every $\sigma \in \Sigma$ there exist $n(\sigma) \in \Sigma$ and finite polyhedra $X_{\sigma}$ and $Y_{\sigma}$ such that the $n$th terms of $\mathbb{X}_{\sigma}$ and $\mathbb{Y}_{\sigma}$ are $\mathbf{S}^{n-n(\sigma)}\left(X_{\sigma}\right)$ and $\mathbf{S}^{n-n(\sigma)}\left(Y_{\sigma}\right)$ for sufficiently large $n$.

Therefore without loss of generality we may assume that the following conditions are satisfied:

(1) $n(\sigma) \leq n(\tau)$ if $\sigma \leq \tau$.

(2) The bonding morphisms of $\underline{\mathbf{X}}$ and $\underline{\mathbf{Y}}$ are induced by maps $p_{\sigma}^{\tau}$ : $\mathbf{S}^{m-n(\tau)}\left(X_{\tau}\right) \rightarrow \mathbf{S}^{m-n(\sigma)}\left(X_{\sigma}\right)$ and $q_{\sigma}^{\tau}: \mathbf{S}^{m-n(\tau)}\left(Y_{\tau}\right) \rightarrow \mathbf{S}^{m-n(\sigma)}\left(Y_{\sigma}\right)$ for sufficiently large $m$.

(3) The morphism $\mathbf{f}_{\sigma}$ is induced by a map $f_{\sigma}: X_{\sigma} \rightarrow Y_{\sigma}$.

Let $M_{\sigma} \supset X_{\sigma} \cup Y_{\sigma}$ be the reduced mapping cylinder of $f_{\sigma}$. We know that the pointed pair $\left(\mathbf{S}\left(M_{\sigma}\right), \mathbf{S}\left(X_{\sigma}\right)\right)$ has the same homotopy type as $\left(M^{\prime}, \mathbf{S}\left(X_{\sigma}\right)\right)$, where $M^{\prime}$ denotes the reduced mapping cylinder of $\mathbf{S}\left(f_{\sigma}\right)$ (see $\left[\mathrm{S}-\mathrm{W}_{2}\right.$, p. 67$]$ ).

For every $\tau>\sigma$ and sufficiently large $m$ we can find a map $\widehat{f_{\sigma}^{\tau}}$ : $\mathbf{S}^{m-n(\tau)}\left(M_{\tau}\right) \rightarrow \mathbf{S}^{m-n(\sigma)}\left(M_{\sigma}\right)$ such that $\widehat{f}_{\sigma}^{\tau}$ restricted to $\mathbf{S}^{m-n(\tau)}\left(X_{\tau}\right)$ (or $\mathbf{S}^{m-n(\tau)}\left(Y_{\tau}\right)$ ) equals $p_{\sigma}^{\tau}$ (respectively $q_{\sigma}^{\tau}$ ). Denote by $L(\sigma)$ the long exact sequence

$$
\begin{aligned}
\ldots \leftarrow \pi_{s}^{k}\left(\mathbf{S}^{m-n(\sigma)}\left(X_{\sigma}\right)\right) \stackrel{i_{\sigma}}{\longleftarrow} \pi_{s}^{k}\left(\mathbf{S}^{m-n(\sigma)}\left(M_{\sigma}\right)\right) \stackrel{j_{\sigma}}{\longleftarrow} \\
\pi_{s}^{k}\left(\mathbf{S}^{m-n(\sigma)}\left(M_{\sigma} / X_{\sigma}\right)\right) \stackrel{\delta_{\sigma}}{\longleftarrow} \pi_{s}^{k-1}\left(\mathbf{S}^{m-n(\sigma)}\left(X_{\sigma}\right)\right) \leftarrow \ldots
\end{aligned}
$$

corresponding to the index $\sigma \in \Sigma$. 
If $\tau>\sigma$ then we have a natural morphism from $L(\sigma)$ to $L(\tau)$ induced by the bonding morphisms and $\widehat{f}_{\sigma}^{\tau}$. The image of $e_{\sigma}$ belonging to one of the components of $L(\sigma)$ is denoted by $e_{\tau}$.

We prove that for every $k$ and every $e_{\sigma} \in \pi_{s}^{k}\left(\mathbf{S}^{m-n(\sigma)}\left(M_{\sigma} / X_{\sigma}\right)\right)$ there exists $\tau>\sigma$ such that $e_{\tau}=0$.

If $j_{\sigma}\left(e_{\sigma}\right) \in \pi_{s}^{k}\left(\mathbf{S}^{m-n(\sigma)}\left(M_{\sigma}\right)\right) \cong \pi_{s}^{k}\left(\mathbf{S}^{m-n(\sigma)}\left(Y_{\sigma}\right)\right)$ represents the trivial element of $\pi_{s}^{k}(\underline{\mathbf{Y}})$ then we can find $\tau>\sigma$ such that $e_{\tau} \in \pi_{s}^{k}\left(\mathbf{S}^{m-n(\tau)}\left(M_{\tau} / X_{\tau}\right)\right)$ is contained in the image of $\pi_{s}^{k-1}\left(\mathbf{S}^{m-n(\tau)}\left(X_{\tau}\right)\right)$ under $\delta_{\tau}$ (i.e. $\left.e_{\tau}=\delta_{\tau}\left(e_{\tau}^{\prime}\right)\right)$. By the exactness of $L(\tau)$ (take greater $\tau$ if necessary) and the fact that $\pi_{s}^{k-1}(\underline{\mathbf{f}}): \pi_{s}^{k-1}(\underline{\mathbf{Y}}) \rightarrow \pi_{s}^{k-1}(\underline{\mathbf{X}})$ is an isomorphism, the element $e_{\tau}$ must represent the trivial element of $\pi_{s}^{k}(\underline{\mathbf{Y}})$ and we may assume that $e_{\tau}=0$.

On the other hand, if $j_{\sigma}\left(e_{\sigma}\right)$ does not represent the trivial element of $\pi_{s}^{k}(\underline{\mathbf{Y}})$, then we can find $\tau_{0}>\sigma$ such that $i_{\tau} j_{\tau}\left(e_{\tau}\right) \neq 0$ for every $\tau>\tau_{0}$, which contradicts the exactness of $L(\tau)$.

We may assume that $\mathbf{S}^{m-n(\sigma)}\left(M_{\sigma}\right)$ and $\mathbf{S}^{m-n(\sigma)}\left(X_{\sigma}\right)$ have the same $r$ skeleton, where $r=m-n(\sigma)-2$. Let $\operatorname{dim} \mathbf{S}^{m-n(\sigma)}\left(M_{\sigma}\right)=n$. Since the stable cohomotopy groups of $\mathbf{S}^{m-n(\sigma)}\left(M_{\sigma} / X_{\sigma}\right)$ are finitely generated we can find a finite sequence $\sigma=\sigma_{0}<\sigma_{1}<\ldots<\sigma_{n-r+1}=\tau$ such that the map $\widehat{f}_{\sigma_{i}}^{\sigma_{i+1}}$ induces a trivial homomorphism of stable cohomotopy groups in dimension $n-i$ for $i=0, \ldots, n-r$.

Lemma 2.1.2 implies that there exists a map

$$
g:\left(\mathbf{S}^{m-n(\tau)}\left(M_{\tau}\right), \mathbf{S}^{m-n(\tau)}\left(X_{\tau}\right)\right) \rightarrow\left(\mathbf{S}^{m-n(\sigma)}\left(M_{\sigma}\right), \mathbf{S}^{m-n(\tau)}\left(X_{\sigma}\right)\right)
$$

such that $g$ is homotopic to $\widehat{f}_{\sigma}^{\tau}$ and $g\left(\mathbf{S}^{m-n(\tau)}\left(M_{\tau}\right)\right) \subset \mathbf{S}^{m-n(\sigma)}\left(X_{\sigma}\right)$. It is known ([Mo, Theorem (1.1)] or [Dy, p. 7, Theorem 2.3]) that this means that $\underline{\mathbf{f}}$ is an isomorphism in Pro- $\mathcal{S C} \mathcal{W}_{f}$.

REMARK 2. If $\underline{\mathbf{X}}=\left\{\operatorname{Sus}\left(X_{\sigma}\right)\right\}$ and $\underline{\mathbf{Y}}=\left\{\operatorname{Sus}\left(Y_{\sigma}\right)\right\}$, then the morphism $\underline{\mathbf{f}}$ is an isomorphism iff it induces isomorphisms of stable cohomotopy groups in dimensions $\geq 0$. In the notation of the proof of Theorem 3.2.1 this means that $n(\sigma)=n(\tau)=0$. Lemma 2.1.2 allows us to prove a stronger version of the theorem in this case.

REMARK 3. The proof of Theorem 3.2.1 is a modification of the classical Whitehead Theorem for the prohomotopy category (see [Dy] and [Ma-Se]).

Theorem 3.2.2. Let $X$ and $Y$ be compact Hausdorff spaces. A morphism $\underline{\mathbf{f}} \in \operatorname{ShStab}(X, Y)$ is an isomorphism iff it induces isomorphisms of stable cohomotopy groups in dimensions $\geq 0$.

Proof. This is a consequence of Theorem 3.2.1 (see also Remark 2). 


\subsection{The Whitehead Theorem for Inj-SCW}

TheOREM 3.3.1. A morphism $\underline{\mathbf{f}} \in \operatorname{Inj-} \mathcal{S C W}_{f}(\underline{\mathcal{A}}, \underline{\mathcal{B}})$ is an isomorphism iff it induces isomorphisms of all stable homotopy groups.

Proof. The morphism $\underline{\mathbf{f}}$ is an isomorphism iff $\mathbf{D}_{2}(\underline{\mathbf{f}}): \mathbf{D}_{2}(\underline{\mathcal{B}}) \rightarrow \mathbf{D}_{2}(\underline{\mathcal{A}})$ is an isomorphism. The assertion is now a consequence of Theorem 3.2.1.

REMARK 4. In [Mi] T. Miyata defined the generalized coshape category $\operatorname{coSh}_{\text {spec }}$, whose objects are $\mathrm{CW}$ spectra and spaces. If $\mathbb{E}$ and $\mathbb{E}$ are $\mathrm{CW}$ spectra, then $\operatorname{coSh}_{\text {spec }}(\mathbb{E}, \mathbb{F})=\langle\mathbb{E}, \mathbb{F}\rangle \cong \operatorname{Inj}_{-} \mathcal{S C} \mathcal{W}_{f}(\underline{\mathbf{E}}, \underline{\mathbf{F}})$, where $\underline{\mathbf{E}}=\left\{\mathbb{E}_{\sigma}\right\}$, $\underline{\mathbf{F}}=\left\{\mathbb{F}_{\tau}\right\} \in \mathrm{ObInj}-\mathcal{S C} \mathcal{W}_{f}$ are direct systems consisting of all finite CW subspectra $\mathbb{E}_{\sigma}$ of $\mathbb{E}$ and (respectively) $\mathbb{E}_{\tau}$ of $\mathbb{E}$ (ordered by inclusion). Miyata stated the Whitehead Theorem for $\operatorname{coSh}_{\text {spec }}$. In particular he claimed that $\varphi \in \operatorname{coSh}_{\text {spec }}(\mathbb{E}, \mathbb{F})$ was an isomorphism of the category $\operatorname{coSh}_{\text {spec }}$ iff it induced isomorphisms of integral homology groups, but this statement is false.

More precisely, it is true that $\varphi \in \operatorname{coSh}_{\mathrm{spec}}(\mathbb{E}, \mathbb{E})$ is an isomorphism when $\varphi$ induces isomorphisms of homotopy groups, but in order to conclude that $\varphi \in \operatorname{coSh}_{\text {spec }}(\mathbb{E}, \mathbb{F})$ is an isomorphism from the fact that it induces isomorphisms of homology groups we must assume that $\mathbb{E}$ and $\mathbb{E}$ are bounded below (see Example 1).

\subsection{The stable shape of the quotient space}

TheOREM 3.4.1. Let $(X, A)$ be a pair of compact Hausdorff spaces. The inclusion of $A$ into $X$ is a stable shape equivalence if and only if $X / A$ has trivial stable shape.

Proof. By Theorem 3.2.2 the inclusion of $A$ into $X$ is a stable shape equivalence if and only if it induces isomorphisms of all stable cohomotopy groups. In the same manner we deduce that $X / A$ has trivial stable shape iff $\pi_{s}^{n}(X / A)=0$ for every $n$.

Consider the long exact sequence

$$
\ldots \rightarrow \pi_{s}^{n-1}(X) \rightarrow \pi_{s}^{n-1}(A) \rightarrow \pi_{s}^{n}(X / A) \rightarrow \pi_{s}^{n}(X) \rightarrow \pi_{s}^{n}(A) \rightarrow \ldots
$$

The groups $\pi_{s}^{n}(X / A)$ are trivial for every $n$ if and only if the inclusion $A \subset X$ induces isomorphisms from $\pi_{s}^{n}(X)$ to $\pi_{s}^{n}(A)$ for every $n$.

\section{FUNCTION SPECTRA}

\subsection{CW substitutes for spectra}

Definition 2. Let $\mathbb{E}$ be a $\mathrm{CW}$ spectrum and $\mathbb{E}$ be a spectrum. A pair $(\mathbb{E}, \boldsymbol{\alpha})$, where $\boldsymbol{\alpha}: \mathbb{E}_{0} \rightarrow \mathbb{E}$ is a function defined on a cofinal subspectrum $\mathbb{E}_{0}$ of $\mathbb{E}$, is called a $C W$ substitute for $\mathbb{E}$ if $\boldsymbol{\alpha}$ is a weak homotopy equivalence. 
When no confusion can arise we ignore the second element of the pair $(\mathbb{E}, \boldsymbol{\alpha})$ and we say that $\mathbb{E}$ is a $\mathrm{CW}$ substitute for $\mathbb{F}$.

This notion is adapted from $[\mathrm{K}-\mathrm{K}-\mathrm{S}]$. In $[\mathrm{K}-\mathrm{K}-\mathrm{S}]$ it is required that $\boldsymbol{\alpha}$ is defined on $\mathbb{E}$ (not on a cofinal subspectrum of $\mathbb{E}$ ), but for our purposes this small modification allows us to simplify notation.

We need the following proposition (Propositions 2.4 and 2.5 of $[\mathrm{K}-\mathrm{K}-\mathrm{S}]$ ).

Proposition 4.1.1. For every spectrum $\mathbb{F}$ there exists a $C W$ substitute of $\mathbb{F}$. If $\boldsymbol{\alpha}: \mathbb{W} \rightarrow \mathbb{E}$ and $\boldsymbol{\beta}: \mathbb{V} \rightarrow \mathbb{F}$ are $C W$ substitutes for spectra $\mathbb{E}$ and $\mathbb{F}$, then for every function $\gamma: \mathbb{E} \rightarrow \mathbb{E}$ there is a unique morphism $\left[\gamma^{\prime}\right]: \mathbb{W} \rightarrow \mathbb{V}$ such that $[\boldsymbol{\gamma}] \circ[\boldsymbol{\alpha}]=[\boldsymbol{\beta}] \circ\left[\boldsymbol{\gamma}^{\prime}\right]$.

REMARK 5. Let the pair $\left(\mathbb{W}_{1}, \boldsymbol{\alpha}\right)$ be a $\mathrm{CW}$ substitute for $\mathbb{E}$ and $\boldsymbol{\beta}$ : $\mathbb{W}_{2} \rightarrow \mathbb{W}_{1}$ be a function between CW spectra. The pair $\left(\mathbb{W}_{2}, \boldsymbol{\alpha} \boldsymbol{\beta}\right)$ is a $\mathrm{CW}$ substitute for $\mathbb{E}$ if $\boldsymbol{\beta}$ represents an isomorphism in the category of spectra. More precisely ([Sw, p. 137]), there are cofinal subspectra $\mathbb{W}_{1}^{\prime} \subset \mathbb{W}_{1}$ and $\mathbb{W}_{2}^{\prime} \subset \mathbb{W}_{2}$ such that $\boldsymbol{\beta}\left(\mathbb{W}_{2}^{\prime}\right) \subset \mathbb{W}_{1}^{\prime}$, the function $\boldsymbol{\alpha}$ is defined on $\mathbb{W}_{1}^{\prime}$ and the composition $\left.\left.\boldsymbol{\alpha}\right|_{\mathbb{W}_{1}^{\prime}} \boldsymbol{\beta}\right|_{\mathbb{W}_{2}^{\prime}}$ is a weak homotopy equivalence.

\subsection{Function spectra}

Definition 3. For every compact Hausdorff space $X$ we will consider the function spectrum $\mathbb{F}(X)=\left(F_{n}, \lambda_{n}\right)$, where $F_{n}=\operatorname{Map}\left(X, S^{n}\right)$ and the maps $\lambda_{n}: \mathbf{S} \operatorname{Map}\left(X, S^{n}\right) \rightarrow \operatorname{Map}\left(X, S^{n+1}\right)$ are defined by $\lambda_{n}(f \wedge t)(x)=$ $f(x) \wedge t$.

Assume that $f: X \rightarrow Y$ is a map. Then for every $n=1,2, \ldots$ we have the $\operatorname{map} f_{n}=\operatorname{Map}(f): \operatorname{Map}\left(Y, S^{n}\right) \rightarrow \operatorname{Map}\left(X, S^{n}\right)$ induced by $f$. The sequence $\mathbb{F}(f)=\left\{f_{n}\right\}: \mathbb{F}(Y) \rightarrow \mathbb{F}(X)$ is a function between spectra.

If $\left(\mathbb{W}_{X}, \boldsymbol{\alpha}_{X}\right)$ and $\left(\mathbb{W}_{Y}, \boldsymbol{\alpha}_{Y}\right)$ are CW substitutes for (respectively) $\mathbb{F}(X)$ and $\mathbb{F}(Y)$, then by Proposition 4.1.1 there is a unique morphism of spectra $\underline{\mathbf{f}}=\left(\mathbb{F}(f), \boldsymbol{\alpha}_{X}, \boldsymbol{\alpha}_{Y}\right)$ such that the diagram

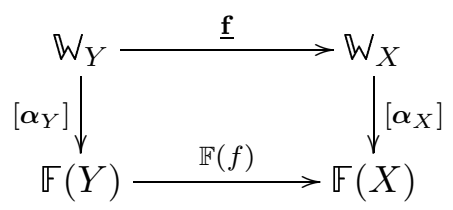

commutes.

Proposition 4.2.1. Let $f: X \rightarrow Y$ and $g: Y \rightarrow Z$ be maps, where $X, Y$ and $Z$ are compact Hausdorff spaces. Then $\underline{\mathbf{h}}=\underline{\mathbf{f g}}$, where $\underline{\mathbf{f}}=$ $\left(\mathbb{F}(f), \boldsymbol{\alpha}_{X}, \boldsymbol{\alpha}_{Y}\right), \underline{\mathbf{g}}=\left(\mathbb{F}(g), \boldsymbol{\alpha}_{Y}, \boldsymbol{\alpha}_{Z}\right)$ and $\underline{\mathbf{h}}=\left(\mathbb{F}(g f), \overline{\boldsymbol{\alpha}}_{X}, \boldsymbol{\alpha}_{Z}\right)$.

Proposition 4.2.2. Let $X$ be a compact Hausdorff space and $(\mathbb{W}, \boldsymbol{\alpha})$ be a $C W$ substitute for $\mathbb{E}(X)$. For every finite $C W$ spectrum $\mathbb{E}$ the function $\boldsymbol{\alpha}$ 
induces an isomorphism

$$
[\mathbb{E}, \mathbb{W}] \rightarrow \underset{\lim }{\longrightarrow}\left\{\ldots \rightarrow\left[E_{n}, \operatorname{Map}\left(X, S^{n}\right)\right] \rightarrow\left[E_{n+1}, \operatorname{Map}\left(X, S^{n+1}\right)\right] \rightarrow \ldots\right\} .
$$

For every $k$ there exists a canonical isomorphism $\pi_{k}(\mathbb{W}) \rightarrow \pi_{s}^{-k}(X)$.

Proof. The first part is obvious (Proposition 1.3.1). The second part is a consequence of the first and elementary properties of spaces of maps from $X$ to spheres. Indeed, $\left[S^{k+l}, W_{n}\right] \cong\left[S^{k+l}, \operatorname{Map}\left(X, S^{n}\right)\right] \cong\left[S^{k+l} \wedge X, S^{n}\right]$ for all $k, l \in \mathbb{Z}$ and every $n=0,1,2, \ldots$

4.3. Complements in spheres and function spaces. Following [K-K-S] for every proper closed subset $X$ of the $(n+1)$-dimensional sphere we introduce a map from $S^{n+1} \backslash X$ to $\operatorname{Map}\left(X, S^{n}\right)$ inducing isomorphisms of homology groups in dimensions $<2(n-\operatorname{dim} X)$. Recall that $S^{0}=\{a, b\} \subset$ $\ldots \subset S^{n} \subset S^{n+1}$.

If $X \ni b$ is a subcompactum of $S^{m}$ which misses $a$ and $n+1 \geq m$, then $\left(S^{n+1} \backslash X\right) \wedge X \subset S^{n+1} \wedge S^{n+1} \backslash D_{n+1} \cong S^{2 n+2} \backslash D_{n+1}$, where $(a, b)$ is the base point of $S^{n+1} \wedge S^{n+1}$ and $D_{n+1}$ denotes the diagonal. The space $D_{n+1}$ is homeomorphic to $S^{n+1}$ and $S^{2 n+2} \backslash D_{n+1}$ contains a polyhedron $T_{n}$ homeomorphic to $S^{n}$ as a strong deformation retract. We may assume that $\left(T_{n+1}, T_{n}\right)$ is homeomorphic to $\left(S^{n+1}, S^{n}\right)$.

Denote by $\varrho_{n}:\left(S^{n+1} \backslash X\right) \wedge X \rightarrow S^{n}$ the composition of the inclusion $\left(S^{n+1} \backslash X\right) \wedge X \subset S^{2 n+2} \backslash D_{n+1}$ with the strong deformation retraction and the canonical homeomorphism of $T_{n}$ onto $S^{n}$. Let

$$
\mu_{n}^{X}: S^{n+1} \backslash X \rightarrow \operatorname{Map}\left(X, S^{n}\right)
$$

be the adjoint to $\varrho_{n}$. Then

(4) if $X \supset Y, a \notin X$ and $b \in Y$, then $\mu_{n}^{X}(z)=\mu_{n}^{Y}(z)$ for every $z \in Y$.

The next proposition can be found in [K-K-S, p. 212].

Proposition 4.3.1. The map $\mu_{n}^{X}: S^{n+1} \backslash X \rightarrow \operatorname{Map}\left(X, S^{n}\right)$ induces an isomorphism $\left(\mu_{n}^{X}\right)_{\star}: H_{q}\left(S^{n+1} \backslash X\right) \rightarrow H_{q}\left(\operatorname{Map}\left(X, S^{n}\right)\right)$ for $q<$ $2(n-\operatorname{dim} X)$.

Let $X$ be a compact subpolyhedron of $S^{n+1}$ with $\operatorname{dim} X<n-1$ and let $M$ be a regular neighborhood of $X$ in $S^{n+1}$. The interior $V=\operatorname{Int} M$ is an open regular neighborhood of $X$ and $S^{n+1} \backslash V$ is a compact subpolyhedron of $S^{n+1}$. Denote by

$$
\mu_{n}^{(M, X)}: S^{n+1} \backslash V \rightarrow \operatorname{Map}\left(X, S^{n}\right)
$$

the composition $S^{n+1} \backslash V \subset S^{n+1} \backslash X \stackrel{\mu_{n}^{X}}{\longrightarrow} \operatorname{Map}\left(X, S^{n}\right)$.

LEMMA 4.3.2. Let $M$ and $N$ be regular neighborhoods of a subpolyhedron $X$ of $S^{n+1}$ with $\operatorname{dim} X<n-1$ such that $W=\operatorname{Int} N \subset N \subset V=\operatorname{Int} M$. 
If $i_{N}^{M}: S^{n+1} \backslash V \rightarrow S^{n+1} \backslash W$ and $r_{X}^{N}: \operatorname{Map}\left(N, S^{n}\right) \rightarrow \operatorname{Map}\left(X, S^{n}\right)$ are (respectively) the inclusion and the restriction map, then:

(i) $r_{X}^{N}$ and $i_{N}^{M}$ are homotopy equivalences.

(ii) The following diagram commutes:

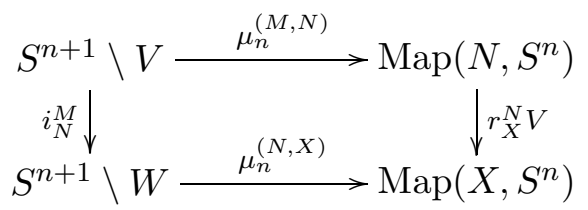

(iii) There is a map $\mu_{n}^{(M, M)}: S^{n+1} \backslash V \rightarrow \operatorname{Map}\left(M, S^{n}\right)$, unique up to homotopy, such that $r_{X}^{M} \mu_{n}^{(M, M)} \simeq \mu_{n}^{(M, X)}$.

Proof. It is clear that $S^{n+1} \backslash X$ is a connected and simply connected open subset of $S^{n+1}$. The spaces $W$ and $V$ are also connected and simply connected. From the Alexander Duality Theorem it follows that the inclusion $i_{N}^{M}$ induces isomorphisms of homology groups. The Whitehead Theorem implies that it must be a homotopy equivalence.

The complements of regular neighborhoods of $X$ are also simply connected and connected. As previously (using the Alexander Duality and the Whitehead Theorem) we find that the inclusions $i_{1}: S^{n+1} \backslash M \rightarrow S^{n+1} \backslash N$, $i_{2}: S^{n+1} \backslash N \rightarrow S^{n+1} \backslash X, j_{1}: S^{n+1} \backslash V \rightarrow S^{n+1} \backslash N$ and $j_{2}: S^{n+1} \backslash W \rightarrow$ $S^{n+1} \backslash X$ are homotopy equivalences.

The restriction maps $r_{N}^{M}: \operatorname{Map}\left(M, S^{n}\right) \rightarrow \operatorname{Map}\left(N, S^{n}\right), r_{X}^{N}: \operatorname{Map}\left(N, S^{n}\right)$ $\rightarrow \operatorname{Map}\left(X, S^{n}\right)$ and $r_{X}^{M}: \operatorname{Map}\left(M, S^{n}\right) \rightarrow \operatorname{Map}\left(X, S^{n}\right)$ are also homotopy equivalences (being induced by inclusions which are homotopy equivalences).

This means that (ii) is satisfied.

Let $\mu_{n}^{(M, M)}=j \mu_{n}^{(M, X)}$, where $j$ denotes the homotopy inverse of $r_{X}^{M}$. Since $\mu_{n}^{(M, X)}$ and $j$ are homotopy equivalences, we infer that $\mu_{n}^{(M, M)}$ is unique up to homotopy.

REMARK 6. Let $X$ be a proper subpolyhedron of $S^{n+1}$ and $M_{k}$ be a regular neighborhood of $X$ in $S^{k}$ for $k \geq n+1$ such that $M_{k+1} \cap S^{k}=M^{k}$ for $k \geq n+1$. Then the Spanier-Whitehead dual of the $\operatorname{spectrum} \operatorname{Sus}(X)$ is isomorphic to the spectrum $\mathbb{W}=\left\{W_{k}\right\}$, where $W_{k}=S^{k+1} \backslash \operatorname{Int} M_{k+1}$ for $k \geq n+1$ (cf. Theorem 4.5 of $[\mathrm{K}-\mathrm{K}-\mathrm{S}]$ ).

Moreover, the maps $\mu_{k}^{\left(M_{k+1}, X\right)}: S^{k+1} \backslash W_{k+1} \rightarrow \operatorname{Map}\left(X, S^{k}\right)$ generate a function $\mu: \mathbf{D}(X)=\mathbf{D}(\operatorname{Sus}(X)) \rightarrow \mathbb{F}(X)$. The pair $(\mathbf{D}(X), \mu)$ is a CW substitute for $\mathbb{E}(X)$.

4.4. Substitutes of function spectra and limits. Let $\mathbb{W}$ be a $\mathrm{CW}$ spectrum. By $\underline{\mathbf{W}} \in \mathrm{Ob} \operatorname{Inj}-\mathcal{S C} \mathcal{W}_{f}$ we denote the direct system consisting of all finite subspectra of $\mathbb{W}$ ordered by inclusion. 
Suppose that a compact Hausdorff space $X$ is the inverse limit of a system $\underline{\mathbf{X}}=\left\{X_{\sigma}, p_{\tau}^{\sigma}, \Sigma\right\}$ of finite CW complexes. We have observed (see Remark 6) that for every $\sigma \in \Sigma$ there exists a function $\mu_{\sigma}: \mathbf{D}\left(\operatorname{Sus}\left(X_{\sigma}\right)\right) \rightarrow$ $\mathbb{F}\left(X_{\sigma}\right)$ which is a weak homotopy equivalence.

The canonical projections $p_{\sigma}: X \rightarrow X_{\sigma}$ and the bonding morphisms $p_{\sigma}^{\tau}$ : $X_{\tau} \rightarrow X_{\sigma}$ induce functions $\mathbf{p}_{\sigma}: \mathbb{F}\left(X_{\sigma}\right) \rightarrow \mathbb{F}(X)$ and $\mathbf{p}_{\sigma}^{\tau}: \mathbb{F}\left(X_{\sigma}\right) \rightarrow \mathbb{F}\left(X_{\tau}\right)$. It is clear that $\mathbf{p}_{\tau} \mathbf{p}_{\sigma}^{\tau}=\mathbf{p}_{\sigma}$.

Let $\left(\mathbb{W}_{X}, \boldsymbol{\alpha}_{X}\right)$ be a CW substitute for $\mathbb{E}(X)$ and let $\boldsymbol{\alpha}_{\sigma}: \mathbf{D}\left(\operatorname{Sus}\left(X_{\sigma}\right)\right) \rightarrow$ $\mathbb{W}_{X}$ be the unique morphism of spectra such the diagram

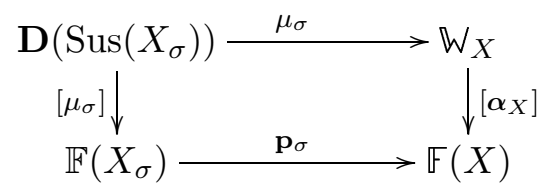

commutes. We know that $\boldsymbol{\alpha}_{\sigma}=\boldsymbol{\alpha}_{\tau} \boldsymbol{\alpha}_{\tau}^{\sigma}$, where $\boldsymbol{\alpha}_{\sigma}^{\tau}: \mathbf{D}\left(\operatorname{Sus}\left(X_{\sigma}\right)\right) \rightarrow$ $\mathbf{D}\left(\operatorname{Sus}\left(X_{\tau}\right)\right)$ is a morphism of spectra induced by $p_{\sigma}^{\tau}: X_{\tau} \rightarrow X_{\sigma}$.

Notice (see Proposition 1.4.2) that $\operatorname{Inj}-\mathcal{S C} \mathcal{W}_{f}\left(\left\{\mathbf{X}_{\sigma}, \boldsymbol{\alpha}_{\sigma}^{\tau}, \Sigma\right\}, \underline{\mathbf{W}}\right)$ can be identified with the direct limit of the system $\left\{\left[\mathbb{K}_{\sigma}, \mathbb{W}\right],\left(\boldsymbol{\alpha}_{\sigma}^{\tau}\right)_{\star}, \Sigma\right\}$, where $\mathbb{W}$ is a $\mathrm{CW}$ spectrum and the function $\left(\boldsymbol{\alpha}_{\sigma}^{\tau}\right)_{\star}:\left[\mathbb{K}_{\tau}, \mathbb{W}\right] \rightarrow\left[\mathbb{K}_{\sigma}, \mathbb{W}\right]$ is induced by $\boldsymbol{\alpha}_{\sigma}^{\tau}$.

TheOREm 4.4.1. Suppose that a compact Hausdorff space $X$ is the inverse limit of the inverse system $\underline{\mathbf{X}}=\left\{X_{\sigma}, p_{\sigma}^{\tau}, \Sigma\right\}$ of finite $C W$ complexes and $\left(\mathbb{W}_{X}, \boldsymbol{\alpha}_{X}\right)$ is a $C W$ substitute for the function spectrum $\mathbb{E}(X)$. Then the morphism $\underline{\boldsymbol{\alpha}}=\left\{\boldsymbol{\alpha}_{\sigma}\right\}:\left\{\mathbf{D}\left(\operatorname{Sus}\left(X_{\sigma}\right)\right), \boldsymbol{\alpha}_{\sigma}^{\tau}, \Sigma\right\} \rightarrow \underline{\mathbf{W}}_{X}$ is an isomorphism in the category Inj-SCW $\mathcal{W}_{f}$.

Proof. We know that the function $\boldsymbol{\alpha}_{X}$ induces an isomorphism $\alpha_{\#}^{k}$ : $\pi_{k}\left(\underline{\mathbf{W}}_{X}\right) \rightarrow \underline{\lim }\left\{\ldots \rightarrow \pi_{k+m}\left(\operatorname{Map}\left(X, S^{m}\right)\right) \rightarrow \pi_{k+m+1}\left(\operatorname{Map}\left(X, S^{m+1}\right)\right) \rightarrow \ldots\right\}$.

By Theorem 3.3.1 it suffices to prove that $\underline{\boldsymbol{\alpha}}$ induces an isomorphism of homotopy groups, i.e. $\underline{\boldsymbol{\alpha}}$ induces a monomorphism and an epimorphism.

Let $\mathbf{e} \in \pi_{k}\left(\underline{\mathbf{W}}_{X}\right)$ and let $\alpha_{\#}^{k}(\mathbf{e})$ be represented by a homotopy class $[f]: S^{k+m} \rightarrow \operatorname{Map}\left(X, S^{m}\right)$. Since $\left[S^{k+m}, \operatorname{Map}\left(X, S^{m}\right)\right]$ is isomorphic to $\lim \left\{\left[S^{k}, \operatorname{Map}\left(X_{\sigma}, S^{m}\right)\right] \cong \lim \left[S^{k} \wedge X_{\sigma}, S^{m}\right] \cong\left[S^{k+m} \wedge X, S^{m}\right]\right.$, we infer that

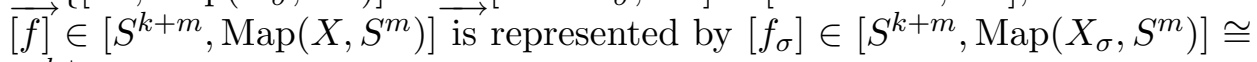
$\left[S^{k+m} \wedge X_{\sigma}, S^{m}\right]$ for sufficiently large $\sigma$. Therefore $\underline{\boldsymbol{\alpha}}$ induces a monomorphism.

The homotopy class $[f] \in\left[S^{k+m}, \operatorname{Map}\left(X, S^{m}\right)\right] \cong\left[X, \operatorname{Map}\left(S^{k+m}, S^{m}\right)\right]$ represents a trivial element of $\pi_{k+m}\left(\operatorname{Map}\left(X, S^{m}\right)\right)$ if and only if $\left[f_{\sigma}\right]$ : $\left[S^{k+m}, \operatorname{Map}\left(X_{\sigma}, S^{m}\right)\right] \cong\left[X_{\sigma}, \operatorname{Map}\left(S^{k+m}, S^{m}\right)\right]$ represents a trivial element of $\pi_{k+m}\left(\operatorname{Map}\left(X_{\sigma}, S^{m}\right)\right)$ for sufficiently large $\sigma$. This means that $\underline{\boldsymbol{\alpha}}$ induces an epimorphism. 
Corollary 4.4.2. Suppose that a compact Hausdorff space $X$ is homeomorphic to the inverse limit of a sequence $\underline{\mathbf{X}}=\left\{X_{\sigma}, p_{\sigma}^{\tau}, \Sigma\right\}$ of finite $C W$ complexes and $\left(\mathbb{W}_{X}, \boldsymbol{\alpha}_{X}\right)$ is a $C W$ substitute for the function spectrum $\mathbb{F}(X)$. Then $\left\{\operatorname{Sus}\left(X_{\sigma}\right), \operatorname{Sus}\left(p_{\sigma}^{\tau}\right), \Sigma\right\}$ and $\mathbf{D}_{2}\left(\underline{\mathbf{W}}_{X}\right)$ are isomorphic objects of Pro- $\mathcal{S C W}$.

Proof. By Theorem 4.4.1 the direct systems $\left\{\mathbf{D}\left(\operatorname{Sus}\left(X_{\sigma}\right)\right), \boldsymbol{\alpha}_{\sigma}^{\tau}, \Sigma\right\}$ and

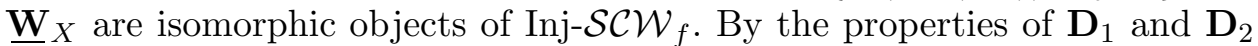
the inverse systems $\left\{\operatorname{Sus}\left(X_{\sigma}\right), \operatorname{Sus}\left(p_{\sigma}^{\tau}\right), \Sigma\right\}$ and $\mathbf{D}_{2}\left(\left\{\mathbf{D}\left(\operatorname{Sus}\left(X_{\sigma}\right)\right), \boldsymbol{\alpha}_{\sigma}^{\tau}, \Sigma\right\}\right)$ are isomorphic as objects of Pro- $\mathcal{S C W} \mathcal{W}_{f}$ and the direct systems $\mathbf{D}_{1} \mathbf{D}_{2}\left(\underline{\mathbf{W}}_{X}\right)$ and $\underline{\mathbf{W}}_{X}$ are isomorphic in Inj-SCW $\mathcal{W}_{f}$. This means that $\mathbf{D}_{2} \mathbf{D}_{1} \mathbf{D}_{2}\left(\underline{\mathbf{W}}_{X}\right) \cong$ $\mathbf{D}_{2}\left(\underline{\mathbf{W}}_{X}\right) \cong\left\{\operatorname{Sus}\left(X_{\sigma}\right), \operatorname{Sus}\left(p_{\sigma}^{\tau}\right), \Sigma\right\}$.

\subsection{Substitutes and suspensions}

THEOREM 4.5.1. Let $X$ be a compact Hausdorff space and $(\mathbb{W}, \boldsymbol{\alpha})$ be a $C W$ substitute for $\mathbb{F}(X)$, where $\boldsymbol{\alpha}=\left\{\alpha_{n}\right\}$. Then $\left(\mathbf{S}^{-1}(\mathbb{W}), \boldsymbol{\beta}\right)$ is a $C W$ substitute for $\mathbb{F}(\mathbf{S}(X))$, where the function $\boldsymbol{\beta}: \mathbf{S}^{-1}(\mathbb{W}) \rightarrow \mathbb{F}(\mathbf{S}(X))$ is given by $\beta_{n}=\lambda_{n} \alpha_{n-1}$ and $\lambda_{n}: \operatorname{Map}\left(X, S^{n-1}\right) \rightarrow \operatorname{Map}\left(\mathbf{S}(X), S^{n}\right)$ is induced by suspension.

Proof. We may assume that $X$ is the inverse limit of an inverse system $\underline{\mathbf{X}}=\left\{X_{\sigma}, p_{\sigma}^{\tau}, \Sigma\right\}$ of finite $\mathrm{CW}$ complexes. Then $\mathbf{S}(X)$ is the inverse limit of the system $\mathbf{S}(\underline{\mathbf{X}})=\left\{\mathbf{S}\left(X_{\sigma}\right), \mathbf{S}\left(p_{\sigma}^{\tau}\right), \Sigma\right\}$ and the assertion is a consequence of Theorems 3.3.1 and 4.4.1.

\section{CLASSES OF SPECTRA WHICH ARE CW SUBSTITUTES OF FUNCTION SPECTRA}

5.1. Representing compacta as limits of inverse sequence of polyhedra lying in spheres. In this section unless otherwise stated it is understood that a proper submanifold $M$ in $S^{n}$ is a PL compact submanifold (with boundary) and $\operatorname{dim} M=n$.

If $M \subset S^{n}$ misses the base point of $S^{n}$ and $[\alpha, \beta] \subset(-1,1)$, then the set $M \times[\alpha, \beta]$ consists of all points $(x, t) \in S^{n+1}$ such that $x \in M$ and $t \in[\alpha, \beta]$.

Definition 4. A sequence $\mathcal{M}=\left\{\left(M_{n}, X_{n}\right), r_{n}\right\}_{n=1}^{\infty}$ is called a defining sequence if the following conditions are satisfied for every $n$ :

(a) $X_{n}$ is a subpolyhedron of $S^{n+1}$ with $3 \operatorname{dim} X_{n}<n$ and $M_{n}$ a proper submanifold in $S^{n+2}$, missing the base point of $S^{n+2}$.

(b) $M_{n}$ is a neighborhood of $X_{n} \cup X_{n+1}$ in $S^{n+2}$, the inclusion of $X_{n}$ into $M_{n}$ is a homotopy equivalence and $r_{n}: M_{n} \rightarrow X_{n}$ is a deformation retraction.

(c) $M_{n} \times[-1 / 2,1 / 2]$ is a neighborhood of $M_{n+1}$ in $S^{n+1}$. 
The inverse sequence $\nabla(\mathcal{M})=\left\{X_{n}, p_{n}^{n+1}\right\}$, where $p_{n}^{n+1}$ is the composition of the inclusion of $X_{n+1}$ into $M_{n}$ and $r_{n}$, is said to be the inverse sequence corresponding to $\mathcal{M}$.

For every $n$ there exists a cellular decomposition of the spectrum $\mathbb{S}$ such that $\mathbb{V}_{n}=\mathbf{S}^{-n-1}\left(\operatorname{Sus}\left(V_{n}\right)\right)$ is a $\mathrm{CW}$ subspectrum of $\mathbb{S}$, where $V_{n}$ is the complement of the interior of $M_{n-1}$ in $S^{n+1}$. The decomposition assigned to $n+1$ is a subdivision of the decomposition assigned to $n$.

Finally, the sequence $\triangle(\mathcal{M})=\left\{V_{n}\right\}=\bigcup_{n=1}^{\infty} \mathbb{V}_{n}$ is a CW spectrum.

To shorten notation we shall write $\left\{\left(M_{n}, X_{n}\right)\right\}_{n=1}^{\infty}$ instead of $\left\{\left(M_{n}, X_{n}\right), r_{n}\right\}_{n=1}^{\infty}$.

LEMMA 5.1.1. For every compact pair $(X, A)$ there exist defining sequences $\mathcal{M}=\left\{\left(M_{n}, X_{n}\right), r_{n}\right\}_{n=1}^{\infty}$ and $\mathcal{N}=\left\{\left(N_{n}, A_{n}\right), \widehat{r}_{n}\right\}_{n=1}^{\infty}$ such that:

(1) $X$ is homeomorphic to the limit of the inverse sequence $\nabla(\mathcal{M})$.

(2) $A$ is homeomorphic to the limit of the inverse sequence $\nabla(\mathcal{N})$.

(3) $N_{n}$ is a subpolyhedron of $M_{n}$ and $X_{n} \supset A_{n}$.

(4) $\widehat{r}_{n}: N_{n} \rightarrow A_{n}$ is an extension of $r_{n}: M_{n} \rightarrow X_{n}$.

Proof. There exists an inverse sequence $\left\{\left(Y_{n}, B_{n}\right), q_{n}^{n+1}\right\}$ of pairs of polyhedra such that the bonding morphisms are PL maps, $3 \operatorname{dim} Y_{n}<n$ and $(X, A)$ is homeomorphic to the inverse limit of $\left\{\left(Y_{n}, B_{n}\right), q_{n}^{n+1}\right\}$. Observe that $Y_{n}$ is 0 -dimensional for $n=1,2,3$. We may also assume that $Y_{1}=Y_{2}$ is a one-point set.

We shall define $\mathcal{M}=\left\{\left(M_{n}, X_{n}\right), r_{n}\right\}_{n=1}^{\infty}$ inductively. We shall also need to define PL homeomorphisms $h_{n}: Y_{n} \rightarrow X_{n}$ such that

$$
h_{n} q_{n}^{n+1} h_{n+1}^{-1}(x)=r_{n}(x) \quad \text { for every } x \in X_{n+1} \text {. }
$$

These conditions are satisfied for $n=1,2$ if $X_{1}=X_{2}$ is a one-point set. The sets $M_{1}$ and $M_{2}$ must be small contractible closed neighborhoods of $X_{1}$. The maps $h_{1}, h_{2}, r_{1}$ and $r_{2}$ are defined automatically and equal to the constant maps.

Assume that $M_{n-1}, X_{n}, h_{n}$ and $r_{n-1}$ are constructed and satisfy (6) for $n<k$. The mapping $M$ cylinder of $q_{k}^{k+1}$ is a polyhedron (see [Ma-Se, p. 295, Theorem 6]) with $3 \operatorname{dim} M<k+2$. By the Unknotting Theorem (see [R, p. 119]) there exists a PL embedding $g: M \rightarrow S^{k+2}$ such that $g(x)=$ $h_{k}(x)$ for $x \in Y_{k} \subset M$. We may assume that $g(M)$ is a subset of the interior of $M_{k} \times[-1 / 2,1 / 2]$. Indeed, a regular neighborhood $N$ of $g(M)$ is a regular neighborhood of $X_{k}$. It follows that there exists a PL isotopy of $S^{k+2}$ which does not move points of $X_{k}$ and transforms $N$ into a subset of $\operatorname{Int}\left(M_{k} \times[-1 / 2,1 / 2]\right)$.

Hence we may find a regular neighborhood $M_{k}$ of $g(M)$ such that $M_{k} \subset$ $\operatorname{Int}\left(M_{k-1} \times[-1 / 2,1 / 2]\right)$. Let $X_{k+1}=g\left(Y_{k+1}\right)$ and let $h_{k+1}: Y_{k+1} \rightarrow X_{k+1}$ be defined by $h_{k+1}(x)=g(x)$ for $x \in Y_{k+1} \subset M$. Clearly, one can find 
a deformation retraction $r_{k}: M_{k} \rightarrow X_{k}$ such that (6) is satisfied for $n<$ $k+1$.

5.2. Substitutes of function spectra of compacta. Assume that $W_{n}$ is a proper submanifold in $S^{n+1}$ such that $\mathbf{S}\left(W_{n}\right) \subset W_{n+1}$ for every $n$. Then $\mathbb{W}=\left\{W_{n}\right\}$ is a $\mathrm{CW}$ spectrum. The cellular decomposition of $W_{n}$ induces a cellular decomposition of $\mathbf{S}\left(W_{n}\right) \subset W_{n+1}$ and one can decompose into cells the closure of $W_{n+1} \backslash \mathbf{S}\left(W_{n}\right)$ in such a way that eventually we get a cellular decomposition of $W_{n+1}$, compatible with the structure of $\mathbf{S}\left(W_{n}\right)$ as a CW complex.

LEMMA 5.2.1. Let $X_{n}$ be a subpolyhedron in $S^{n+1}$ and let $W_{n}, V_{n}, M_{n-1}$ be proper submanifolds of $S^{n+1}$ such that:

(1) $\mathbf{S}\left(W_{n}\right) \subset V_{n+1} \subset W_{n+1}$ and the first inclusion is a homotopy equivalence.

(2) $M_{n} \subset S^{n+2} \backslash V_{n+1}$ and the inclusion is a homotopy equivalence.

(3) The inclusions of $X_{n} \subset M_{n}$ and $X_{n} \subset S^{n+1} \backslash W_{n}$ are homotopy equivalences.

(4) $M_{n}$ is a neighborhood of $X_{n+1} \cup X_{n}$ in $S^{n+2}$.

Then $\mathbf{D}_{1}(\underline{\mathbf{X}}) \cong \underline{\mathbf{W}}$ and $\mathbf{D}_{2}(\underline{\mathbf{W}}) \cong \underline{\mathbf{X}}$, where $\underline{\mathbf{X}}=\left\{\operatorname{Sus}\left(X_{n}\right), \mathbf{p}_{n}^{n+1}\right\} \in$ Pro-SCW $\mathcal{W}_{f}$. The morphism $\mathbf{p}_{n}^{n+1}: \operatorname{Sus}\left(X_{n+1}\right) \rightarrow \operatorname{Sus}\left(X_{n}\right)$ is induced by the composition of the inclusion $X_{n+1} \subset M_{n}$ and the deformation retraction $r: M_{n} \rightarrow X_{n}$. The $C W$ spectrum $\mathbb{W}_{n}$ is defined by $\mathbb{W}_{n}=\mathbf{S}^{-1}\left(\operatorname{Sus}\left(W_{1}\right)\right) \cup$ $\mathbf{S}^{-2}\left(\operatorname{Sus}\left(W_{2}\right)\right) \cup \ldots \cup \mathbf{S}^{-n}\left(\operatorname{Sus}\left(W_{n}\right)\right)$ and the morphism $\mathbf{i}_{n}^{n+1}: \mathbb{W}_{n} \rightarrow \mathbb{W}_{n+1}$ is induced by the inclusion $\mathbf{S}\left(W_{n}\right) \subset W_{n+1}$. The inclusions $\mathbf{S}\left(W_{n}\right) \subset V_{n+1}$ induce an isomorphism of $\underline{\mathbf{V}}$ onto $\underline{\mathbf{W}}$.

Proof. Without loss of generality (see Remark 1) we may assume that $\mathbf{D}\left(\operatorname{Sus}\left(X_{n}\right)\right)=\mathbb{W}_{n}, \mathbf{D}\left(\operatorname{Sus}\left(X_{n+1}\right)\right)=\mathbb{W}_{n+1}, \mathbf{D}\left(\operatorname{Sus}\left(M_{n}\right)\right)=\mathbf{S}^{-n}\left(\operatorname{Sus}\left(W_{n}\right)\right)$ and the images under $\mathbf{D}$ of the morphisms induced by the inclusions $\operatorname{Sus}\left(X_{n}\right) \subset \operatorname{Sus}\left(M_{n}\right)$ and $\operatorname{Sus}\left(X_{n+1}\right) \subset \operatorname{Sus}\left(M_{n}\right)$ are equal to the morphisms induced by the inclusions $\mathbf{S}^{-n}\left(\operatorname{Sus}\left(W_{n}\right)\right) \subset \mathbb{W}_{n}$ and $\mathbf{S}^{-n}\left(\operatorname{Sus}\left(W_{n}\right)\right) \subset \mathbb{W}_{n+1}$. Hence $\mathbf{D}\left(\mathbf{p}_{n}^{n+1}\right)=\mathbf{i}_{n}^{n+1}$ and $\mathbf{D}_{1}(\underline{\mathbf{X}}) \cong \underline{\mathbf{W}}$.

Proposition 5.2.2. If $\mathcal{M}=\left\{\left(M_{n}, X_{n}\right)\right\}_{n=1}^{\infty}$ is a defining sequence, then $\mathbf{S}(\triangle(\mathcal{M}))$ is a $C W$ substitute for the spectrum $\mathbb{E}(X)$, where $X$ is the inverse limit of the sequence $\underline{\mathbf{X}}=\nabla(\mathcal{M})$.

Proof. This is a consequence of Theorems 4.4.1, 4.5.1 and Lemma 5.2.1.

Corollary 5.2.3. For every compactum $X$ there exists a countable $C W$ substitute $\mathbb{W}_{X}$ for $\mathbb{F}(X)$.

COROLlary 5.2.4. Let $\mathbb{W}$ be a $C W$ spectrum. If a compactum $X$ is homeomorphic to the limit of a sequence $\left\{X_{k}, p_{k}^{k+1}\right\}$ of finite $C W$ complexes and 
$\left\{\mathbf{D}\left(\operatorname{Sus}\left(X_{k}\right)\right), \mathbf{D}\left(\operatorname{Sus}\left(p_{k}^{k+1}\right)\right)\right\}$ is isomorphic to $\underline{\mathbf{W}}$, then $\mathbb{W}$ is a $C W$ substitute for $\mathbb{E}(X)$.

Proof. By the previous lemma there exists a CW substitute $\mathbb{W}_{X}$ for $\mathbb{F}(X)$. Corollary shows 4.4 .2 that the direct sequences $\mathbb{W}_{X}$ and $\mathbb{W}$ are isomorphic objects of Inj-SCW $\mathcal{W}_{f}$.

Since $\mathbb{W}_{X}$ is a countable spectrum we infer (see Proposition 1.4.3) that any isomorphism from $\mathbb{W}_{X}$ to $\mathbb{W}$ must be represented by a function of spectra (defined on a cofinal subspectrum of $\mathbb{W}_{X}$ ). This map generates an isomorphism of $\mathcal{S C W}$.

\subsection{Spectra which are CW substitutes for function spectra}

Proposition 5.3.1. For $n=1,2, \ldots$, let $W_{n}$ be a proper submanifold in $S^{n+1}$ such that $\mathbf{S}\left(W_{n}\right) \subset W_{n+1}$. Then there exists a compactum $X$ such that $\mathbb{W}=\left\{W_{n}\right\}$ is a $C W$ substitute for $\mathbb{E}(X)$.

Proof. Let $X_{1}, \ldots, X_{k}$ be polyhedra in $S^{n+1}$ and for each $i=1, \ldots, k$ let $C_{i}$ be a compact subset of $S^{n+1} \backslash X_{i}$. There are polyhedra $Y_{1}, \ldots, Y_{k}$ such that $C_{i} \subset Y_{i} \subset S^{n+1} \backslash X_{i}$ and $Y_{i}$ is a deformation retract of $S^{n+1} \backslash X_{i}$ and whenever $X_{i} \subset Y_{j}$ then $Y_{j} \subset Y_{i}$ (see [S-W $\left.\mathrm{W}_{1}, \mathrm{p} .58\right]$ ). Using this fact we can construct sequences $X_{n}, M_{n}, W_{n}$ and $V_{n}$ satisfying conditions (1)-(4) of Lemma 5.2.1.

Proposition 5.3.2. Let $\mathbb{W}=\left\{W_{n}\right\}$ be a $C W$ spectrum such that each $W_{n}$ is a finite $C W$ complex which can be embedded in $S^{n}$. Then there exists a shape 1-connected continuum $X$ such that $\mathbf{S}^{-1}(\mathbb{W})$ is a $C W$ substitute for $\mathbb{E}(X)$.

Proof. The space $S^{n+2} \backslash W_{n}=O_{n}$ has the homotopy type of a finite simply connected and connected CW complex $X_{n}$ (see [W, p. 67]). Let $h_{n}$ : $O_{n} \rightarrow X_{n}$ be a homotopy equivalence. The inclusion $i: O_{n} \rightarrow S^{n+3} \backslash \mathbf{S}\left(W_{n}\right)$ is a homotopy equivalence. Indeed, by the Alexander Duality Theorem it induces isomorphisms of singular homology groups.

Let $j: S^{n+3} \backslash W_{n+1} \rightarrow S^{n+3} \backslash \mathbf{S}\left(W_{n}\right)$ denote the inclusion, let $r_{1}$ : $S^{n+3} \backslash \mathbf{S}\left(W_{n}\right) \rightarrow O_{n}$ and $r_{2}: X_{n+1} \rightarrow S^{n+3} \backslash W_{n+1}$ be homotopy inverses for (respectively) $i$ and $h_{n+1}$, and set $p_{n}^{n+1}=h_{n} r_{1} j r_{2}: X_{n+1} \rightarrow X_{n}$.

It follows by the same method as in Proposition 5.3.1 that the inverse limit $X$ of the sequence $\left\{X_{n}, p_{n}^{n+1}\right\}$ satisfies the required conditions.

\subsection{Spectra bounded below with no cells in dimensions $>0$}

THEOREM 5.4.1. Suppose that $\mathbb{W}$ is a countable spectrum bounded below with no cells in dimensions $>0$ and $\pi_{j}(\mathbb{W})=0$ for $j<-m<0$. Then there exists a finite-dimensional compactum $X$ such that $\mathbf{S}^{-m-1}(\mathbb{W})$ is a $C W$ substitute for $\mathbb{E}(X)$. 
Proof. There exists a filtration $\mathbb{Y}_{1} \subset \mathbb{Y}_{2} \subset \ldots$ of $\mathbb{W}$ into finite subspectra. Consider the inverse sequence $\underline{\mathbf{X}}=\left\{\mathbb{X}_{n}, \boldsymbol{\alpha}_{n}^{n+1}\right\}$, where $\boldsymbol{\alpha}_{n}^{n+1}: \mathbb{X}_{n+1}=$ $\mathbf{D}\left(\mathbb{Y}_{n+1}\right) \rightarrow \mathbf{D}\left(\mathbb{Y}_{n}\right)=\mathbb{X}_{n}$ is the Spanier-Whitehead dual for the inclusion of $\mathbb{Y}_{n}$ into $\mathbb{Y}_{n+1}$. It is clear (see Theorem 3.1.1) that $\pi^{j}(\underline{\mathbf{X}})=0$ for $j>-m$ and $\pi_{i}\left(\mathbb{X}_{n}\right)=0$ for $i \leq 0$.

We may assume that for every $n$ there exist an index $\sigma(n)>m+1$, a finite connected CW complex $X_{n}$ and a map $\widehat{p}_{n}^{n+1}: X_{n+1} \rightarrow \mathbf{S}^{a(n)}\left(X_{n}\right)$, where $a(n)=\sigma(n+1)-\sigma(n)$, such that the following conditions are satisfied:

- $\sigma(n+1)>\sigma(n)$,

- $\boldsymbol{\alpha}_{n}^{n+1}$ is induced by $\widehat{p}_{n}^{n+1}$,

- $\pi^{\sigma(n)-j}\left(\mathbb{Y}_{n}\right)=\pi_{j}\left(X_{n}\right)=\pi_{j}^{s}\left(X_{n}\right)=0$ for $j=0,1, \ldots, \sigma(n)-1$.

The vanishing of the stable cohomotopy groups of $\underline{\mathbf{X}}$ for dimensions $>m$ implies that the proof can be reduced to the case when $\widehat{p}_{n}^{n+1}: X_{n+1} \rightarrow$ $\mathbf{S}^{a(n)}\left(X_{n}\right)$ induces a trivial homomorphism of stable cohomotopy groups in dimensions $>m+\sigma(n+1)$. Indeed, the stable cohomotopy groups of a finite CW complex are finitely generated and we can replace $\underline{\mathbf{X}}$ by a suitable subsequence.

It follows from Lemma 2.1.2 that for sufficiently large $k$ there exist $n^{\prime}>n$ and a map $q: \mathbf{S}^{k}\left(X_{n^{\prime}}\right) \rightarrow \mathbf{S}^{k+\sigma\left(n^{\prime}\right)-\sigma(n)}\left(X_{n}\right)$ whose stable homotopy class is the same as that of $p_{n}^{n^{\prime}}: X_{n^{\prime}} \rightarrow \mathbf{S}^{\sigma\left(n^{\prime}\right)-\sigma(n)}\left(X_{n}\right)$ and $q\left(X_{n^{\prime}}\right)$ is contained in the $\left(m+k+\sigma\left(n^{\prime}\right)-\sigma(n)\right)$-skeleton of $\mathbf{S}^{k+\sigma\left(n^{\prime}\right)-\sigma(n)}\left(X_{n}\right)$.

This means that choosing a suitable subsequence of $\underline{\mathbf{X}}$ we may reduce the proof to the case when $\widehat{p}_{n}^{n+1}\left(X_{n}\right) \subset \mathbf{S}^{a(n)}\left(V_{n}\right)$, where $V_{n}$ is the $(m+\sigma(n))$ skeleton of $X_{n}$.

Let $\underline{\mathbf{V}}=\left\{\mathbf{S}^{-\sigma(n)}\left(\operatorname{Sus}\left(V_{n}\right)\right)\right\} \in$ ObPro- $\mathcal{S C} \mathcal{W}_{f}$. We denote by $\widehat{q}_{n}^{n+1}$ : $V_{n+1} \rightarrow \mathbf{S}^{a(n)}\left(V_{n}\right)$ the restriction of $\widehat{p}_{n}^{n+1}$ to $V_{n+1}$ and by $\mathbf{i}_{n}: \mathbf{S}^{-\sigma(n)}\left(\operatorname{Sus}\left(V_{n}\right)\right)$ $\rightarrow \mathbb{X}_{n}$ the morphism of spectra induced by the inclusion $V_{n} \subset X_{n}$. Theorem 3.2.1 implies that $\underline{\mathbf{i}}=\left\{\mathbf{i}_{n}\right\} \in \operatorname{Pro}-\mathcal{S C W}_{f}(\underline{\mathbf{V}}, \underline{\mathbf{X}})$ is an isomorphism.

Since $V_{n}$ is a $(\sigma(n)-1)$-connected finite $\mathrm{CW}$ complex with dimension $<m+\sigma(n)$ we infer that there exist a finite $\mathrm{CW}$ complex $W_{n}$ and a homotopy equivalence $f_{n}: \mathbf{S}^{\sigma(n)-m-1}\left(W_{n}\right) \rightarrow V_{n}$ such that $\operatorname{dim} W_{n} \leq 2 m$ (see $\left[\mathrm{S}_{2}\right.$, p. 461, Exercise D1]).

The Freudenthal Suspension Theorem implies that there exists a map $q_{n}^{n+1}: W_{n+1} \rightarrow W_{n}$ such that $\left[\mathbf{S}^{\sigma(n+1)-m-1}\left(q_{n}^{n+1}\right)\right]=\left[\mathbf{S}^{a(n)}\left(f_{n}\right)\right]\left[\widehat{q}_{n}^{n+1}\right]\left[g_{n+1}\right]$, where $g_{n}$ denotes the homotopy inverse of $f_{n}$ for every $n$ (see the diagram).

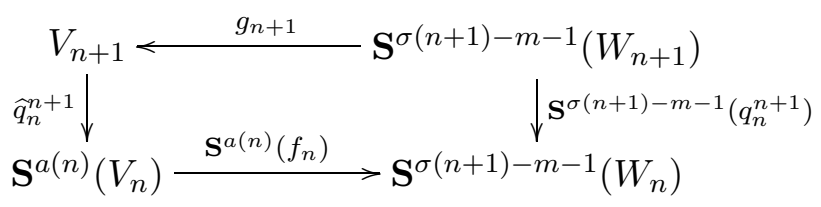


Let $\underline{\mathbf{W}}=\left\{\mathbf{S}^{-m-1}\left(\operatorname{Sus}\left(W_{n}\right)\right)\right\} \in$ ObPro-SCW$f$ and let $\underline{\mathbf{g}}=\left\{\mathbf{g}_{n}\right\} \in$ Pro- $\mathcal{S C} \mathcal{W}_{f}(\underline{\mathbf{V}}, \underline{\mathbf{W}})$ be a morphism, where $\mathbf{g}_{n}$ denotes the morphism of spectra induced by $g_{n}: V_{n} \rightarrow \mathbf{S}^{\sigma(n)-m-1}\left(W_{n}\right)$. It follows from Theorem 3.2.1 that $\underline{\mathrm{g}}$ is an isomorphism. Hence $\underline{\mathbf{i g}}: \mathbf{S}^{-m-1}(\underline{\mathbf{W}}) \rightarrow \underline{\mathbf{X}}$ is also an isomorphism, $\overline{\mathbf{D}}_{1}\left(\mathbf{S}^{-m-1}(\underline{\mathbf{W}})\right) \cong \mathbf{D}_{1}(\underline{\mathbf{X}}) \cong \bar{W}$ and $\left.\mathbf{D}_{1}(\underline{\mathbf{W}})\right) \cong \mathbf{S}^{-m-1}(\mathbb{W})$.

By Theorem 4.4.1, we infer (Remark 5) that $\mathbf{S}^{(-m-1)}(\mathbb{W})$ is a CW substitute for $\mathbb{F}(X)$, where $X$ is the inverse limit of the inverse sequence $\left\{W_{n}, q_{n}^{n+1}\right\}$.

COROllary 5.4.2. For every countable $C W$ complex $W$ with $\operatorname{dim} W \leq n$ and $-m>2 n+2$ there exists a continuum $X$ such that $\mathbf{S}^{m}(\operatorname{Sus}(W))$ is a $C W$ substitute for $\mathbb{E}(X)$.

\section{DUALITY}

\subsection{Main theorems}

TheOREM 6.1.1. For every pair $X, Y$ of compact Hausdorff spaces and every pair $\left(\mathbb{W}_{X}, \boldsymbol{\alpha}\right),\left(\mathbb{W}_{Y}, \boldsymbol{\beta}\right)$ of $C W$ substitutes for (respectively) $\mathbb{E}(X)$ and $\mathbb{F}(Y)$ there exists an isomorphism $\mathcal{D}_{\left(\mathbb{W}_{X}, \mathbb{W}_{Y}\right)}^{X, Y}=\mathcal{D}: \operatorname{ShStab}(X, Y) \rightarrow\left\langle\mathbb{W}_{Y}, \mathbb{W}_{X}\right\rangle$ such that:

(i) If $\left(\mathbb{V}_{X}, \boldsymbol{\alpha}^{\prime}\right)$ and $\left(\mathbb{V}_{Y}, \boldsymbol{\beta}^{\prime}\right)$ are also $C W$ substitutes for (respectively) $\mathbb{F}(X)$ and $\mathbb{E}(Y)$ then the diagram

$$
\begin{gathered}
\mathbb{W}_{Y} \stackrel{\mathcal{D}_{\left(\mathbb{W}_{X}, \mathbb{W}_{Y}\right)}^{X,(\underline{\mathbf{f}})}}{\longrightarrow} \mathbb{W}_{X} \\
\left\langle\xi_{X}\right\rangle \downarrow \\
\mathbb{V}_{Y} \stackrel{\mathcal{D}_{\left(\mathbb{V}_{X}, \mathbb{V}_{Y}\right)}^{X, \mathbf{f})} \underset{\downarrow}{\longrightarrow}}{\longrightarrow} \mathbb{V}_{X}
\end{gathered}
$$

commutes for every stable shape morphism $\underline{\mathbf{f}} \in \operatorname{ShStab}(X, Y)$, where $\boldsymbol{\xi}_{X}$ : $\mathbb{W}_{X} \rightarrow \mathbb{V}_{X}$ and $\boldsymbol{\xi}_{Y}: \mathbb{W}_{Y} \rightarrow \mathbb{V}_{Y}$ are canonical (unique) isomorphisms of spectra.

(ii) $\mathcal{D}(\underline{\mathbf{g}} \circ \underline{\mathbf{f}})=\mathcal{D}(\underline{\mathbf{f}}) \circ \mathcal{D}(\underline{\mathbf{g}})$, where $\underline{\mathbf{f}} \in \operatorname{ShStab}(X, Y), \underline{\mathbf{g}} \in \operatorname{ShStab}(Y, Z)$.

(iii) $\mathcal{D}\left(\underline{\mathbf{i}}_{X}\right)=\left\langle\underline{\mathbf{i}}_{\mathbb{W}_{X}}\right\rangle$, where $\underline{\mathbf{i}}_{X} \in \operatorname{ShStab}(X, X)$ and $\underline{\mathbf{i}}_{\mathbb{W}_{X}}$ denote respectively the stable shape morphism induced by the identity map of $X$ and the morphism $\underline{\mathbf{i}}_{\mathbb{W}_{X}}: \mathbb{W}_{X} \rightarrow \mathbb{W}_{X}$ induced by the identity of $\mathbb{W}_{X}$.

(iv) $h_{Y}^{n}(X) \cong h_{X}^{n}(Y)$, where $\left\{h_{Y}^{n}\right\}$ and $\left\{h_{X}^{n}\right\}$ denote (respectively) the cohomology theories associated with $\mathbb{W}_{Y}$ and $\mathbb{W}_{X}$.

(v) If $Y$ is a compactum, then $h_{\mathbb{E}}^{n}(X \wedge Y) \cong h_{\mathbb{E} \wedge \mathbb{W}_{Y}}^{n}(X)$, where $\left\{h_{\mathbb{E}}^{n}\right\}$ and $\left\{h_{\mathbb{E} \wedge \mathbb{W}_{Y}}^{n}\right\}$ denote the cohomology theories associated with $\mathbb{E}$ and $\mathbb{E} \wedge \mathbb{W}_{Y}$.

Proof. We have the duality isomorphism (see Definition 1) $\mathbf{D}_{1}$ : $\operatorname{Pro}-\mathcal{S C} \mathcal{W}_{f}(\underline{\mathbf{X}}, \underline{\mathbf{Y}}) \rightarrow \operatorname{Inj}-\mathcal{S C W} \mathcal{W}_{f}\left(\mathbf{D}_{1}(\underline{\mathbf{Y}}), \mathbf{D}_{1}(\underline{\mathbf{X}})\right)$ which is natural with respect to morphisms of Pro- $\mathcal{S C} \mathcal{W}_{f}$. There also exist (see Theorem 4.4.1) 
canonical isomorphisms $\chi_{X}: \mathbf{D}_{1}(\underline{\mathbf{X}}) \rightarrow \underline{\mathbf{W}}_{X}$ and $\chi_{Y}: \mathbf{D}_{1}(\underline{\mathbf{Y}}) \rightarrow \underline{\mathbf{W}}_{Y}$. Let $\mathcal{D}_{\left(\mathbb{W}_{X}, W_{Y}\right)}^{X, Y}(\underline{\mathbf{f}})=\mathcal{D}(\underline{\mathbf{f}}) \chi_{Y} \mathbf{D}_{1}(\underline{\mathbf{f}}) \chi_{X}^{-1}$ for every $\underline{\mathbf{f}} \in \operatorname{ShStab}(X, Y)$.

Observe that $h_{Y}^{n}(X) \cong\left(h_{Y}\right)_{-n}\left(\mathbb{W}_{X}\right) \cong\left(h_{X}\right)_{-n}\left(\mathbb{W}_{Y}\right) \cong h_{X}^{n}(Y)$ (see $\left[\mathrm{A}_{1}\right.$, p. 198]).

It remains to prove $(\mathrm{v})$ in the case when $X$ is a finite $\mathrm{CW}$ complex (see [Sw, pp. 255 and pp. 268]). We may also assume that $Y$ is the inverse limit of a sequence $\left\{Y_{k}, q_{k}^{k+1}\right\}$ of finite $\mathrm{CW}$ complexes and that there exists a filtration $\mathbb{W}_{1} \subset \mathbb{W}_{2} \subset \ldots$ of $\mathbb{W}_{Y}$ such that $\mathbb{W}_{k}$ is a finite spectrum and $\mathbf{D}\left(\operatorname{Sus}\left(Y_{k}\right)\right)=\mathbb{W}_{k}$ for every $k$. Then $h_{\mathbb{E}}^{n}(X \wedge Y) \cong \lim _{\mathbb{E}} h_{\mathbb{E}}^{n}\left(X \wedge Y_{k}\right) \cong$ $\stackrel{\lim }{\longrightarrow} h_{\mathbb{E} \wedge \mathbb{W}_{k}}^{n}(X) \cong h_{\mathbb{E} \wedge \mathbb{W}_{Y}}^{n}(X)$.

REMARK 7. There are close connections and similarities between the results of $[\mathrm{Mi}]$ and $\left[\mathrm{Mi}_{-} \mathrm{Se}_{2}\right]$ and Theorem 6.1.1. Generally speaking, [Mi] contains a version of the duality described in Theorem 6.1.1. It is mainly restricted to metrizable compact spaces. Some facts there are almost the same (for example the assertions (i) of Theorem 4.1 from [Mi] and (iv) of Theorem 6.1.1), others are sharper and limited to narrower classes of objects.

In $\left[\mathrm{Mi}-\mathrm{Se}_{2}\right]$ the authors study the stable shape category of all compact Hausdorff spaces and assign to such a space $X$ a generalized homology theory $\mathfrak{H}_{*}$. Next, using a representation theorem, they consider the CW spectrum $E$ representing $\mathfrak{H}_{*}$. In this way they construct a full embedding of the category ShStab into the category of CW spectra with weak homotopy classes as morphisms.

We believe that this construction gives the same result as described in Theorem 6.1.1 when $\mathfrak{C}^{s}(X), \mathfrak{C}^{s}(Y)<\infty$ or when the stable cohomotopy groups of $Y$ are countable.

REMARK 8. In the light of Theorem 6.1.1, the Whitehead Theorem for stable shape 3.2.2 corresponds to the assertion that a natural transformation from a generalized cohomology theory $\mathfrak{H}^{*}=\left\{h^{n}\right\}$ to a generalized cohomology theory $\mathfrak{K}^{*}=\left\{k^{n}\right\}$ is a natural equivalence iff it induces isomorphisms from $h^{n}\left(S^{0}\right)$ to $k^{n}\left(S^{0}\right)$ for every $n$.

Hence Theorem 3.2.2 can be obtained as a corollary of the above fact. Notice also that the proof of Theorem 4.4.1 requires Theorem 3.3.1, which is dual to Theorem 3.2.1.

COROLlary 6.1.2. For every compact Hausdorff space $X$ and every $C W$ substitute $\mathbb{W}_{X}$ for $\mathbb{E}(X)$ there exists an isomorphism $\mathcal{A}_{q}: \pi^{q}(X) \rightarrow \pi_{-q}\left(\mathbb{W}_{X}\right)$ such that the diagram

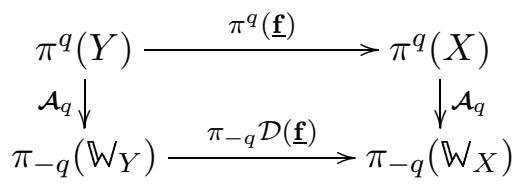

commutes for every $q$. 
Corollary 6.1.3. If $X, Y$ are compact Hausdorff space such that $\mathfrak{C}^{s}(X), \mathfrak{C}^{s}(Y)<\infty$ and $\mathbb{W}_{X}, \mathbb{W}_{Y}$ are $C W$ substitutes for $\mathbb{F}(X), \mathbb{F}(Y)$, then the canonical homomorphism from the group $\left[\mathfrak{W}_{Y}, \mathbb{W}_{X}\right]_{w}$ to $\left\langle\mathbb{W}_{Y}, \mathbb{W}_{X}\right\rangle$ is an isomorphism.

Proof. The spectra $\mathbb{W}_{X}$ and $\mathbb{W}_{Y}$ are bounded below if and only if $\mathfrak{C}^{s}(X)$, $\mathfrak{C}^{s}(Y)<\infty$. By Proposition 1.4.3 we get $\left[\mathbb{W}_{Y}, \mathbb{W}_{X}\right]_{w} \cong\left\langle\mathbb{W}_{Y}, \mathbb{W}_{X}\right\rangle$.

\subsection{Compacta with $\mathfrak{C}^{s}(X)<\infty$}

TheOREm 6.2.1. Let $X$ be a compactum. Then the following conditions are equivalent:

(i) $\mathfrak{C}^{s}(X)<\infty$.

(ii) There exist a finite-dimensional $C W$ complex $W_{X}$ and $l \in \mathbb{Z}$ such that $\mathbf{S}^{l}\left(\operatorname{Sus}\left(W_{X}\right)\right)$ is a $C W$ substitute for $\mathbb{E}(X)$.

(iii) There exist a $C W$ complex $W_{X}$ and $l \in \mathbb{Z}$ such that $\mathbf{S}^{l}\left(\operatorname{Sus}\left(W_{X}\right)\right)$ is a $C W$ substitute for $\mathbb{E}(X)$.

(iv) There exist a $C W$ spectrum $\mathbb{W}_{X}$ bounded below which is a $C W$ substitute for $\mathbb{E}(X)$.

Proof. If $\max \left\{n: \pi_{s}^{n}(X) \neq 0\right\}=N<\infty$, then there exists a compactum $Y$ such that $\operatorname{ShStab}(Y)=\operatorname{ShStab}\left(\mathbf{S}^{N+2}(X)\right)$ and $\operatorname{dim} Y \leq N+2$ (see Lemma 2.3.1). We may assume that $Y \subset S^{8 N}$. Let $W_{X}=S^{8 N} \backslash Y$. Then the spectrum $\mathbb{W}_{X}=\mathbf{S}^{-8 N+1}\left(\operatorname{Sus}\left(W_{X}\right)\right)$ is a CW substitute for $\mathbb{F}(Y)$ (see $[\mathrm{K}-\mathrm{K}-\mathrm{S}])$. On the other hand, $\mathbf{S}^{3-6 N}\left(\mathbb{W}_{X}\right)$ is a CW substitute for $\mathbb{F}(X)$. Hence (i) implies (ii).

It is obvious that (ii) implies (iii) and (iii) implies (iv).

It is known that $\pi_{s}^{n}(X) \cong \pi_{-n}\left(\mathbb{W}_{X}\right)$. Therefore if $\mathbb{W}_{X}$ is bounded below, then $\pi_{s}^{q}(X)=0$ for sufficiently large $q$.

By $\mathcal{C}^{N}$ we denote the class of all compacta $X$ with $\mathfrak{C}^{s}(X) \leq N \in \mathbb{Z}$. By the Menger-Nöbeling Theorem for every $X \in \widetilde{C}^{N}$ there exists a stable shape isomorphism $\boldsymbol{\xi}_{X}: \mathbf{S}^{N+2}(X) \rightarrow \widetilde{X}$, where $\widetilde{X}$ is a closed subset of $S^{4 N+5}$ with $\operatorname{dim} \widetilde{X} \leq 2(N+2)$.

Corollary 6.2.2. Suppose that $\boldsymbol{\xi}_{X}: \mathbf{S}^{N+2}(X) \rightarrow \widetilde{X}$ and $\boldsymbol{\xi}_{Y}: \mathbf{S}^{N+2}(Y)$ $\rightarrow \widetilde{Y}$ are stable shape isomorphisms, where $X, Y \in \mathcal{C}^{N}$ and $\widetilde{X}, \widetilde{Y}$ are closed subsets of $S^{4 N+5}$. Then there exists an isomorphism

$$
\mathcal{D}_{\left(\xi_{X}, \xi_{Y}\right)}^{X, Y}=\mathcal{D}: \operatorname{ShStab}(X, Y) \rightarrow\left\{S^{4 N+5} \backslash \tilde{Y}, S^{4 N+5} \backslash \tilde{X}\right\}_{w}
$$

such that the following conditions are satisfied:

(i) $\mathcal{D}(\mathbf{g} \circ \mathbf{f})=\mathcal{D}(\mathbf{f}) \circ \mathcal{D}(\mathbf{g})$, where $\mathbf{f} \in \operatorname{ShStab}(X, Y)$ and $\mathbf{g} \in \operatorname{ShStab}(Y, Z)$. 
(ii) $\mathcal{D}\left(\mathbf{i}_{X}\right)=\left\{i_{S^{4 N+5} \backslash \tilde{X}}\right\}_{w}$, where $\mathbf{i}_{X} \in \operatorname{ShStab}(X, X)$ and $i_{S^{4 N+5} \backslash \tilde{X}}:$ $S^{4 N+5} \backslash \widetilde{X} \rightarrow S^{4 N+5} \backslash \widetilde{X}$ denote respectively the stable shape morphism induced by the identity map of $X$ and the identity map of $S^{4 N+5} \backslash \widetilde{X}$.

\subsection{Stable shape classification of continua with one nontrivial reduced cohomology group}

Proposition 6.3.1. If $X$ and $Y$ are continua with $\mathfrak{C}^{s}(X), \mathfrak{C}^{s}(Y)<\infty$ and $H^{q}(X ; \mathbb{Z})=0=H^{q}(Y ; \mathbb{Z})$ for every $0<q \neq n$, then $\operatorname{ShStab}(X)=$ $\operatorname{ShStab}(Y)$ if and only if $H^{n}(X ; \mathbb{Z}) \cong H^{n}(Y ; \mathbb{Z})$.

Proof. Clearly $H^{n}(X ; \mathbb{Z}) \cong H^{n}(Y ; \mathbb{Z})$ if $\operatorname{ShStab}(X)=\operatorname{ShStab}(Y)$.

Conversely, suppose that $H^{n}(X ; \mathbb{Z}) \cong H^{n}(Y ; \mathbb{Z}) \cong G$. If $\operatorname{ShStab}\left(\mathbf{S}^{k}(X)\right)$ $=\operatorname{ShStab}\left(\mathbf{S}^{k}(X)\right)$ for some $k$, then $\operatorname{ShStab}(X)=\operatorname{ShStab}(Y)$ (see Proposition 1.4.1). Hence we can reduce the proof to the case when $\operatorname{dim} X=n=$ $\operatorname{dim} Y$ (see Lemma 2.3.1). We embed $X$ and $Y$ into the sphere $S^{2 n+1} \subset S^{m}$. For sufficiently large $m$ the complements $S^{m} \backslash X$ and $S^{m} \backslash Y$ are simply connected ANR's with vanishing reduced homology groups in dimensions $\neq l=m-n-1$. The Alexander Duality Theorem also implies that $H_{l}\left(S^{m} \backslash X ; \mathbb{Z}\right) \cong H_{l}\left(S^{m} \backslash Y ; \mathbb{Z}\right)$.

We may replace (up to homotopy) $X$ and $Y$ by CW complexes $P$ and $Q$ with $\operatorname{dim} P, \operatorname{dim} Q \leq l+1$ ([W, p. 62]). There are CW complexes $\widehat{P}$ and $\widehat{Q}$ such that $\pi_{l}(\widehat{P}) \cong G \cong \pi_{l}(\widehat{Q}), \pi_{i}(\widehat{P})=0=\pi_{i}(\widehat{Q})$ for $i \neq l$ and $\widehat{P}^{(l+1)}=P$, $\widehat{Q}^{(l+1)}=Q$. This shows that there is a cellular map $\widehat{f}: \widehat{P} \rightarrow \widehat{Q}$ such that $\pi_{i}(\widehat{f}): \pi_{i}(\widehat{P}) \rightarrow \pi_{i}(\widehat{Q})$ is an isomorphism for every $i$.

Setting $g(x)=\widehat{f}(x)$ for every $x \in P$ we define a map $g: P \rightarrow Q$ such that $\pi_{l}(g): \pi_{l}(P) \rightarrow \pi_{l}(Q)$ is an isomorphism.

From the Hurewicz Theorem $\left(\left[\mathrm{S}_{2}\right.\right.$, p. 390]) we conclude that $H_{l}(g)$ : $H_{l}(P ; \mathbb{Z}) \rightarrow H_{l}(Q ; \mathbb{Z})$ is an isomorphism and finally that $g$ is a homotopy equivalence.

We now apply Corollary 6.2.2 to deduce that there is an $\underline{\mathbf{f}} \in \operatorname{ShStab}(X, Y)$ which is an isomorphism.

6.4. Movable compacta. Suppose that a compactum $Y$ is the inverse limit of a sequence $\left\{Y_{n}, q_{n}^{n+1}\right\}$, where $Y_{n}$ is a polyhedron for every $n$. Following $\left[\mathrm{Mi}_{-} \mathrm{Se}_{1}\right]$, we say that $Y$ is stable movable if $\underline{\mathbf{Y}}=\left\{\operatorname{Sus}(Y), \operatorname{Sus}(q)_{n}^{n+1}\right\}$ is a movable object ([Ma-Se, p. 159]) of Pro- $\mathcal{S C W}$.

THEOREM 6.4.1. If $Y$ is a stable movable compactum, then the canonical projection of $\left[\mathbb{W}_{Y}, \mathbb{W}_{X}\right]$ onto $\left[\mathbb{W}_{Y}, \mathbb{W}_{X}\right]_{w}$ is an isomorphism.

Proof. Let $h_{k}^{\mathrm{St}}(Y)$ denote the $k$ th Steenrod homology group of $Y$ (see $[\mathrm{K}-\mathrm{K}-\mathrm{S}]$ ) corresponding to the reduced generalized homology theory associated with the spectrum $\mathbb{W}_{X}$. It is known (see [K-K-S, p. 203]) that 
$h_{0}^{\mathrm{St}}(Y)=\left[\mathbb{W}_{Y}, \mathbb{W}_{X}\right]$. We also have $($ see $[\mathrm{K}-\mathrm{K}-\mathrm{S}])$ the exact sequence

$$
0 \rightarrow \lim ^{1} h_{1}^{\mathrm{St}}\left(Y_{n}\right) \rightarrow h_{0}^{\mathrm{St}}(Y) \rightarrow \underset{\lim }{\longleftarrow} h_{0}\left(Y_{n}\right) \rightarrow 0 .
$$

Proposition 1.3.1 implies that $\lim h_{0}\left(Y_{n}\right)=\left[\mathbb{W}_{Y}, \mathbb{W}_{X}\right]_{w}$. If $Y$ is a stable movable compactum, then $\lim { }^{1} h_{1}^{\overleftarrow{S t}}\left(Y_{n}\right)$ is a trivial group (see [Ma-Se, p. 165 and pp. 173]). Hence the canonical projection from $\left[\mathbb{W}_{Y}, \mathbb{W}_{X}\right]$ to $\left[\mathbb{W}_{Y}, \mathbb{W}_{X}\right]_{w}$ is an isomorphism.

\section{COMPACT SPACES HAVING METRIZABLE OR POLYHEDRAL REPRESENTATIVES UP TO STABLE SHAPE}

\subsection{Compact spaces having countable CW substitutes for $\mathbb{E}(X)$}

THEOREM 7.1.1. The following conditions are equivalent:

(a) There exists a countable $C W$ spectrum $\mathbb{W}$ which is a $C W$ substitute for $\mathbb{E}(X)$.

(b) There exists a compact metric space $Y$ such that $\operatorname{ShStab}(X)=$ $\operatorname{ShStab}(Y)$.

(c) The stable cohomotopy groups of $X$ are countable.

Proof. (a) $\Rightarrow$ (b). We can assume that $X$ is the inverse limit of a system $\underline{\mathbf{X}}=\left\{X_{\sigma}, p_{\tau}^{\sigma}, \Sigma\right\}$ of finite CW complexes. Let $\left(\mathbb{W}, \boldsymbol{\alpha}_{X}\right)$ be a CW substitute for $\mathbb{E}(X)$, where $\mathbb{W}$ is a countable spectrum and $\boldsymbol{\alpha}_{X}: \mathbb{W} \rightarrow \mathbb{F}(X)$ is a function of spectra. There exists a filtration $\mathbb{W}_{1} \subset \mathbb{W}_{2} \subset \ldots$ of $\mathbb{W}$ such that $\mathbb{W}_{i}$ is a finite CW spectrum for $i=1,2, \ldots$

Consider the inverse sequence $\underline{\mathbf{Y}}=\left\{\mathbb{Y}_{n}, \mathbf{q}_{n}^{n+1}\right\}$, where $\mathbf{D}\left(\mathbb{W}_{i}\right)=\mathbb{Y}_{i}$ and the bonding morphisms are duals to the inclusions of $\mathbb{W}_{i}$ into $\mathbb{W}_{i+1}$. The sequence $\underline{\mathbf{Y}}$ is isomorphic as an object of Pro- $\mathcal{S C} \mathcal{W}_{f}$ to the inverse system $\underline{\widehat{\mathbf{X}}}=\left\{\operatorname{Sus}\left(X_{\sigma}\right), \mathbf{p}_{\tau}^{\sigma}, \Sigma\right\}$, where $\mathbf{p}_{\tau}^{\sigma}$ denotes the morphism of spectra induced by $p_{\tau}^{\sigma}$ (see Theorem 3.1.1).

Let $\underline{\hat{\mathbf{f}}}=\left(\phi, \widehat{\mathbf{f}}_{n}\right): \underline{\widehat{\mathbf{X}}} \rightarrow \mathbb{Y}$ be an isomorphism and $\underline{\widehat{\mathbf{g}}}=\left(\phi, \widehat{\mathbf{g}}_{\sigma}\right): \underline{\mathbf{Y}} \rightarrow \underline{\widehat{\mathbf{X}}}$ be an inverse for $\underline{\widehat{\mathbf{f}}}$, where $\phi: \mathbb{N} \rightarrow \Sigma, \psi: \Sigma \rightarrow \mathbb{N}, \widehat{\mathbf{f}}_{n}: \mathbb{X}_{\phi(n)} \rightarrow \mathbb{Y}_{n}$ and $\widehat{\mathbf{g}}_{\sigma}: \mathbb{Y}_{\psi(\sigma)} \rightarrow \mathbb{X}_{\sigma}$. Using induction one can show that for every $n \in \mathbb{N}$ there exist morphisms $\mathbf{f}_{n}: \mathbb{K}_{\sigma(n)} \rightarrow \mathbb{Y}_{a(n)}$ and $\mathbf{g}_{n}: \mathbb{Y}_{a(n+1)} \rightarrow \mathbb{X}_{\sigma(n)}$ such that

$$
\mathbf{f}_{n} \mathbf{g}_{n}=\mathbf{q}_{a(n)}^{a(n+1)}, \quad \mathbf{g}_{n} \mathbf{f}_{n}=\mathbf{p}_{\sigma(n)}^{\sigma(n+1)}
$$

where $\sigma(n)<\sigma(n+1)$ and $a(n)<a(n+1)$.

Let $\sigma(1)=\phi(1), \mathbf{f}_{1}=\widehat{\mathbf{f}}_{1}, a(1)=1$. Then there exists $a(2)>\psi(1)$ such that $\mathbf{q}_{a(1)}^{a(2)}=\mathbf{f}_{k} \mathbf{g}_{\sigma(1)} \mathbf{q}_{\psi(\sigma(1))}^{a(2)}$. Let $\mathbf{g}_{1}=\mathbf{g}_{\sigma(1)} \mathbf{q}_{\psi(\sigma(1))}^{a(2)}$.

Suppose that $\mathbf{f}_{k}: \mathbb{X}_{\sigma(k)} \rightarrow \mathbb{Y}_{a(k)}$ and $\mathbf{g}_{k}: \mathbb{Y}_{a(k+1)} \rightarrow \mathbb{X}_{\sigma(k)}$ have been constructed. Then there exist $\sigma(k+1) \in \Sigma$ and $a(k+2) \in \mathbb{N}$ such that 
$\mathbf{g}_{k} \widehat{\mathbf{f}}_{a(k+1)} \mathbf{p}_{\phi(a(k+1))}^{\sigma(k+1)}=\mathbf{p}_{\sigma(k)}^{\sigma(k+1)}$ and $\widehat{\mathbf{f}}_{a(k+1)} \widehat{\mathbf{g}}_{\phi(a(k+1))} \mathbf{q}_{\psi(a(k+1))}^{a(k+2)}=\mathbf{q}_{a(k+1)}^{a(k+2)}$. Let $\mathbf{f}_{k+1}=\widehat{\mathbf{f}}_{a(k+1)} \mathbf{p}_{\phi(a(k+1))}^{\sigma(k+1)}$ and $\mathbf{g}_{k+1}=\widehat{\mathbf{g}}_{\phi(a(k+1))} \mathbf{q}_{\psi(a(k+1))}^{a(k+2)}$. This means that (7) is satisfied for every $n$ (see the diagram).

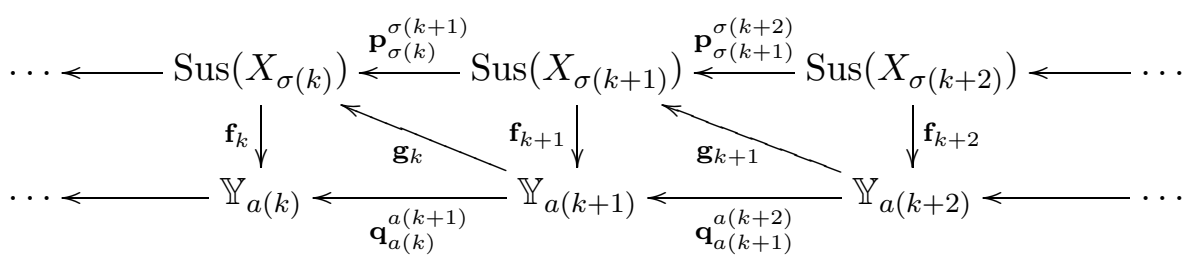

The inverse sequence $\underline{\widetilde{\mathbf{X}}}=\left\{\operatorname{Sus}\left(X_{\sigma(n)}\right), \mathbf{p}_{\sigma(n)}^{\sigma(n+1)}\right\}$ is a subsystem of $\underline{\widehat{\mathbf{X}}}$ and $\pi_{s}^{m}(\underline{\widetilde{\mathbf{X}}}) \cong \pi_{s}^{m}(\underline{\mathbf{Y}}) \cong \pi_{s}^{m}(\underline{\widetilde{\mathbf{X}}})$ for every $m$. Moreover, the restriction morphism from $\underline{\widehat{X}}$ to $\underline{\widetilde{X}}$ induces isomorphisms of stable cohomotopy groups in all dimensions.

Theorem 3.2.1 implies that the restriction morphism is an isomorphism and the inverse limit $Y$ of the sequence $\underline{\widetilde{X}}$ is a compactum with the same stable shape as $X$.

$(b) \Rightarrow(c)$. The stable cohomotopy groups are invariants of the stable shape. It is also clear that the stable cohomotopy groups of a compactum are countable.

$(\mathrm{c}) \Rightarrow(\mathrm{a})$. Suppose that the stable cohomotopy groups of $X$ are countable and the pair $(\mathbb{E}, \boldsymbol{\kappa})$ is a $\mathrm{CW}$ substitute for $\mathbb{F}(X)$, where $\mathbb{E}=\left\{E_{n}, \varepsilon_{n}\right\}$ is an $\Omega_{0}$-spectrum (see $\left[\mathrm{A}_{1}, \mathrm{p}\right.$. 150] and Remark 5) and $\kappa=\left\{\kappa_{n}\right\}$. Since $\pi_{r}\left(E_{n}\right) \cong \pi_{r-n}(\mathbb{E})$ for $r=1,2, \ldots\left(\left[\mathrm{A}_{1}\right.\right.$, p. 134] $)$, we infer that there are countable $\mathrm{CW}$ complexes $F_{n}$ and homotopy equivalences $f_{n}: F_{n} \rightarrow E_{n}$ ([L-W, p. 137, Theorem 6.1]).

Consider the map $\delta_{n}=f_{n+1}^{-1} \varepsilon_{n} \mathbf{S}\left(f_{n}\right): \mathbf{S}\left(F_{n}\right) \rightarrow F_{n+1}$, where $f_{n+1}^{-1}$ is a homotopy inverse of $f_{n+1}$. Replacing $E_{n}$ by the telescope $W_{n}$ of the sequence of maps $\delta_{n}, \mathbf{S}\left(\delta_{n-1}\right), \mathbf{S}^{2}\left(\delta_{n-2}\right), \ldots, \mathbf{S}^{n-1}\left(\delta_{1}\right)$ we get (see [Sw, p. 134, Proposition 8.3]) a CW spectrum $\mathbb{W}=\left\{W_{n}\right\}$ and homotopy equivalences $g_{n}: W_{n} \rightarrow F_{n}$. The properties of the telescope construction guarantee that $\mathbb{W}$ is countable. The diagram

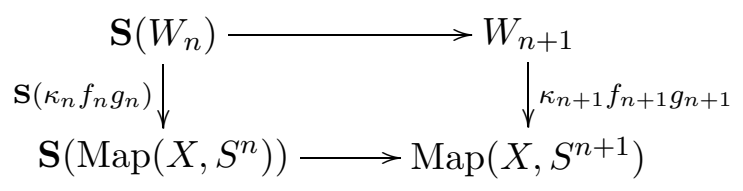

commutes up to homotopy.

By $\left[\mathrm{Sw}\right.$, Lemma 10.4, p. 173]) there exists a function $\boldsymbol{\alpha}=\left\{\alpha_{n}\right\}: \mathbb{W} \rightarrow$ $\mathbb{F}(X)$ such that $\alpha_{n} \simeq \kappa_{n} f_{n} g_{n}: W_{n} \rightarrow \operatorname{Map}\left(X, S^{n}\right)$. The pair $(\mathbb{W}, \boldsymbol{\alpha})$ is a $\mathrm{CW}$ substitute of $\mathbb{F}(X)$. 
REMARK 9. T. Watanabe [Wa] characterized topological spaces $X$ which have the shape of compact metric spaces. The existence of a compactum $Y$ with the same shape as $X$ is equivalent to countability of $[X, P]$ for every finite CW complex $P$. From this he deduced that $X$ has the shape of a compactum if and only if $X$ is shape dominated by a compactum.

Corollary 7.1.2. A compact Hausdorff space $X$ has the stable shape of a compactum if and only if there exists a compactum $Y$ such that $\operatorname{ShStab}(X)$ $\leq \operatorname{ShStab}(Y)$.

Proof. If $\operatorname{ShStab}(X) \leq \operatorname{ShStab}(Y)$, then $\pi_{s}^{n}(Y) r$-dominates $\pi_{s}^{n}(X)$ and (see [Bo, p. 18]) the group $\pi_{s}^{n}(X)$ is a direct summand of $\pi_{s}^{n}(Y)$. Hence, if $\pi_{s}^{n}(Y)$ is countable, then $\pi_{s}^{n}(X)$ is countable.

Corollary 7.1.3. Let $X, Y$ be compact Hausdorff spaces and $\mathbb{W}_{X}, \mathbb{W}_{Y}$ be $C W$ substitutes for $\mathbb{F}(X), \mathbb{F}(Y)$. If the stable cohomotopy groups of $Y$ are countable, then the canonical homomorphism from the group $\left[W_{Y}, \mathbb{W}_{X}\right]_{w}$ to $\left\langle W_{Y}, W_{X}\right\rangle$ is an isomorphism.

Proof. This is a consequence of Theorem 7.1.1 and Proposition 1.4.3.

7.2. Integral cohomology of $X$ with $\mathfrak{C}^{s}(X)<\infty$. It is known $([\mathrm{Hu}$, Chapter X, p. 298]) that there exists a canonical homomorphism $\mathrm{h}^{n}: \pi_{s}^{n}(X) \rightarrow H^{n}(X ; \mathbb{Z})$.

Let $\mathcal{C}$ be a collection of Abelian groups. $\mathcal{C}$ is called a class of Abelian groups if it satisfies the following conditions:

(1) If the sequence $0 \rightarrow G_{1} \rightarrow G_{2} \rightarrow G_{3} \rightarrow 0$ is exact, then $G_{2}$ is in $\mathcal{C}$ if and only if $G_{1}$ and $G_{3}$ are in $\mathcal{C}$.

(2) If $G$ is in $\mathcal{C}$, then $H_{r}(G ; \mathbb{Z})$ is in $\mathcal{C}$.

A homomorphism $f: G \rightarrow H$ is a $\mathcal{C}$-isomorphism if $\operatorname{Ker} f$ and Coker $f$ are in $\mathcal{C}$.

THEOREM 7.2.1. Suppose that $X$ is a compact Hausdorff space with $\mathfrak{C}^{s}(X)<\infty$. Then $\mathfrak{C}^{s}(X)=\max \left\{n: H^{n}(X ; \mathbb{Z}) \neq 0\right\}$ and for every class $\mathcal{C}$ of Abelian groups the following two conditions are equivalent:

(i) The stable cohomotopy groups $\pi_{s}^{k}(X)$ are in $\mathcal{C}$ for $k>n$.

(ii) The cohomology groups $H^{k}(X ; \mathbb{Z})$ are in $\mathcal{C}$ for $k>n$.

Furthermore, either implies that $\mathrm{h}^{n}: \pi_{s}^{n}(X) \rightarrow H^{n}(X ; \mathbb{Z})$ is a $\mathcal{C}$-isomorphism.

Proof. The dual $\mathbb{W}=\mathcal{D}(X)$ of $X$ with $\mathfrak{C}^{s}(X)<\infty$ is a spectrum bounded below. For the class of spectra bounded below one can develop Serre's $\mathcal{C}$-theory. In particular ([Ma, p. 91]), if $\mathbb{W}$ is bounded below and either $\pi_{r}(\mathbb{W})$ is in $\mathcal{C}$ for $r<m$ or $H_{r}(\mathbb{W} ; \mathbb{Z})$ is in $\mathcal{C}$ for $r<m$ then the Hurewicz homomorphism $\mathrm{h}_{r}: \pi_{r}(\mathbb{W}) \rightarrow H_{r}(\mathbb{W} ; \mathbb{Z})$ is a $\mathcal{C}$-isomorphism for $r \leq m$. 
The Hurewicz homomorphism $\mathrm{h}_{r}: \pi_{r}(\mathbb{W}) \rightarrow H_{r}(\mathbb{W} ; \mathbb{Z})$ corresponds to $\mathrm{h}^{-r}: \pi_{s}^{-r}(X) \rightarrow H^{-r}(X ; \mathbb{Z})$ under the duality described in Theorem 6.1.1.

ExAmple 1. Let us denote by $X$ the Adams-Kahn continuum (see $\left[\mathrm{A}_{2}\right]$, $[\mathrm{K}]$ and [Ma-Se, p. 153]). We know that $\mathfrak{C}^{s}(X)=\mathfrak{C}_{s}(X)=\infty$ and $\max \{n$ : $\left.H^{n}(X ; \mathbb{Z}) \neq 0\right\}=0$. If $\mathbb{W}_{X}$ is a $\mathrm{CW}$ substitute for the function spectrum $\mathbb{F}(X)$, then the homology groups $H_{k}\left(\mathbb{W}_{X}\right)$ are the same as the homology of the trivial spectrum (i.e. the suspension spectrum of the singleton). Hence the constant map of $\mathbb{W}_{X}$ into the trivial spectrum induces isomorphisms of homology groups. The spectrum $\mathbb{W}_{X}$ has $\pi_{k}\left(\mathbb{W}_{X}\right) \neq 0$ for infinitely many $k \in \mathbb{Z}$. It follows that the constant map does not induce isomorphisms of homotopy groups. It is also clear that the stable cohomotopy groups of $\mathbb{W}_{X}$ are trivial (compare [Li]).

Corollary 7.2.2. Let $X$ and $Y$ be compacta such that $\mathfrak{C}^{s}(X), \mathfrak{C}^{s}(Y)$ $<\infty$. Then $\mathfrak{C}^{s}(X \wedge Y), \mathfrak{C}^{s}(X \times Y)<\infty$.

Corollary 7.2.3. Let $X$ be a compact Hausdorff space such that $\mathfrak{C}^{s}(X)<\infty$. Then the following conditions are equivalent:

(i) There exists a compact metric space $Y$ such that $\operatorname{ShStab}(X)=$ $\operatorname{ShStab}(Y)$.

(ii) $H^{n}(X ; \mathbb{Z})$ are countable for every $n$.

Proof. See Exercise A1 from Chapter X of [Hu].

Corollary 7.2.4. Let $X$ be a compact Hausdorff space such that $\mathfrak{C}^{s}(X)<\infty$. Then the following conditions are equivalent:

(i) There exists a finite $C W$ complex $P$ with the same stable shape as $\mathbf{S}^{k}(X)$, for some $k \geq 0$.

(ii) $H^{n}(X ; \mathbb{Z})$ is finitely generated for every $n$.

Corollary 7.2.5. Let $X$ and $Y$ be compact Hausdorff spaces such that $\mathfrak{C}^{s}(X), \mathfrak{C}^{s}(X)<\infty$. A stable shape morphism $\underline{\mathbf{f}}: X \rightarrow Y$ is an isomorphism iff $H^{n}(\underline{\mathbf{f}}): H^{n}(Y ; \mathbb{Z}) \rightarrow H^{n}(X ; \mathbb{Z})$ is an isomorphism for every $n$.

\section{APPLICATIONS, PROBLEMS AND FINAL REMARKS}

8.1. Generalized cohomological dimension. Let $\mathfrak{H}=\left\{\mathbf{h}^{n}\right\}$ be a generalized cohomology theory defined on the category $\mathcal{H C W}$ f and satisfying the condition

$$
\mathbf{d} \mathfrak{H}=\max \left\{n: h^{n}\left(S^{0}\right) \neq 0\right\}=0 .
$$

The notion of cohomological dimension with respect to a connected spectrum $\mathbb{E}$ was introduced in $\left[\mathrm{D}_{1}\right]$. We formulate the definition of generalized dimension in terms of the cohomology theory $\mathfrak{H}$ (represented by $\mathbb{E}$ ) instead 
of $\mathbb{E}$. Roughly speaking the connectedness of $\mathbb{E}$ corresponds to the condition $\mathbf{d} \mathfrak{H}=0$.

Let $n$ be a natural number. A compactum $X$ has cohomological dimension at most $n$ with respect to $\mathfrak{H}(\mathfrak{H}-\operatorname{dim} X \leq n)$ if for every closed subset $A$ of $X$ the inclusion $i: A \rightarrow X$ induces an epimorphism $h^{m}(i): h^{m}(X) \rightarrow h^{m}(A)$ for every $m \geq n$. If it is not true that $\mathfrak{H}$-dim $X \leq n$, then $\mathfrak{H}$-dim $X>n$. If $\mathfrak{H}$-dim $X>n$ for every $n$, then $\mathfrak{H}$ - $\operatorname{dim} X=\infty$.

A. N. Dranishnikov $\left(\left[\mathrm{D}_{1}\right],\left[\mathrm{D}_{2}\right]\right)$ also studied the cohomotopical dimension $\boldsymbol{\pi}$-dim, i.e. the generalized cohomological dimension with respect to the stable cohomotopy theory. In particular he has proved that $\pi$-dim $X=\operatorname{dim} X$ for every finite-dimensional $X$, and $\pi$-dim $X=\infty$ if $X$ is strongly infinitedimensional or $X$ is a C-compactum. In $\left[\mathrm{D}_{2}\right]$ an example of a strongly infinite-dimensional compactum $Y$ with $\operatorname{dim}_{\mathbb{Z}} Y=3$ is described.

The next theorem (see $\left[\mathrm{D}_{1}\right.$, equivalence (3) and p. 250]) is analogous to the classical fact of the cohomological dimension theory and can be obtained in a similar way.

THEOREM 8.1.1. For every natural number $n$ the following conditions are equivalent:

(1) $\mathfrak{H}-\operatorname{dim} X \leq n$.

(2) The inclusion $i: A \rightarrow X$ induces an epimorphism $h^{n}(i): h^{n}(X) \rightarrow$ $h^{n}(A)$ for every closed subset $A$ of $X$.

(3) $h^{n+1}(X / A)=0$ for every closed subset $A$ of $X$.

TheOREM 8.1.2. Let $\mathfrak{H}$ be a generalized cohomology theory and $X$ be a compactum. Then:

(i) $\mathfrak{H}-\operatorname{dim} X \leq \boldsymbol{\pi}$-dim $X$.

(ii) If $\operatorname{dim}_{\mathbb{Z}} X<\mathfrak{H}$-dim $X$ then $\boldsymbol{\pi}$-dim $X=\infty$.

(iii) If $Y$ is a compactum and $\mathfrak{H}-\operatorname{dim}(X \times Y)=\infty$ then $\pi$ - $\operatorname{dim} X=\infty$ or $\boldsymbol{\pi}$-dim $Y=\infty$.

Proof. We may assume that $\mathfrak{H}$ is represented by a CW spectrum $\mathbb{E}_{\mathfrak{H}}=$ $\left\{E_{n}\right\}$ such that $E_{n}$ is $n$-connected for every $n$. Then (i) and (ii) are consequences of Corollary 2.3.5 and Theorem 7.2.1, while (iii) is a consequence of Corollary 7.2.2.

8.2. Problems. Suppose that $P$ and $Q$ are connected and simply connected CW complexes. A map $f: P \rightarrow Q$ is a homotopy equivalence iff it induces isomorphisms of homology groups with integer coefficients. It follows easily that $f$ is a homotopy equivalence iff it is a stable homotopy equivalence.

Analogously if $X$ and $Y$ are shape 1-connected finite-dimensional continua then a shape morphism $\underline{\mathbf{f}}: X \rightarrow Y$ is a shape equivalence iff it induces 
isomorphisms of all Čech cohomology groups with coefficients in $\mathbb{Z}$. The last condition holds if and only if $\underline{\mathbf{f}}$ is a stable shape equivalence.

Problem 1. Let $X$ and $Y$ be shape 1-connected continua (or more generally Hausdorff continua). Is it true that a shape morphism $\mathbf{f}: X \rightarrow Y$ is an isomorphism when it induces isomorphisms of stable cohomotopy groups?

Problem 2. Let $X$ be a shape 1-connected continuum with all stable cohomotopy groups vanishing. Is it true that $X$ has trivial shape?

In $[\mathrm{H}] \mathrm{H}$. W. Henn considered (apart from the category ShStab) the category StabSh with the same class of objects as ShStab and with the morphisms defined by the formula

$$
\begin{aligned}
& \operatorname{StabSh}(X, Y) \\
& \left.\quad=\underline{\lim } \operatorname{Sh}(X, Y) \rightarrow \operatorname{Sh}(\mathbf{S}(X), \mathbf{S}(Y)) \rightarrow \operatorname{Sh}\left(\mathbf{S}^{2}(X), \mathbf{S}^{2}(Y)\right) \rightarrow \ldots\right\} .
\end{aligned}
$$

There exists a homomorphism $\underline{\mathrm{H}}: \operatorname{StabSh}(X, Y) \rightarrow \operatorname{ShStab}(X, Y)$ preserving the compositions of morphisms. Henn proved that $\underline{H}$ is an isomorphism when the space $X$ is finite-dimensional.

Problem 3. Is $\underline{\mathrm{H}}$ an isomorphism when $\mathfrak{C}^{s}(X)<\infty$ ?

Problem 4. Let $X$ and $Y$ be shape 1-connected continua. Is it true that $\mathbf{f} \in \operatorname{Sh}(X, Y)$ is an isomorphism iff $\underline{\mathrm{H}}(\mathbf{f})$ is an isomorphism?

Problem 5. Let $X$ be a compact Hausdorff space with $\mathfrak{C}^{s}(X)<\infty$. Do there exist a finite-dimensional compact space $Y$ and an index $l$ such that $Y$ has the same stable shape as the $l$-fold suspension of $X$ ?

Problem 6. Is it true that for every compact Hausdorff space $X$ there exist an integer $N$ and a $\mathrm{CW}$ substitute $\mathbb{W}_{X}$ for $\mathbb{E}(X)$ with no cells in dimensions $>N$ ?

Problem 7. Let $X$ be a compact Hausdorff with $\mathfrak{C}^{s}(X)<\infty$. Do there exist a CW complex $W$ and an index $l$ such that $\mathbf{S}^{l}(\operatorname{Sus}(W))$ is a CW substitute for $\mathbb{E}(X)$ ?

An affirmative answer to Problem 6 would imply an affirmative answer to Problem 7.

Problem 8. Let $\mathbb{E}$ be a $\mathrm{CW}$ spectrum with no cells of dimensions $>0$. Under what conditions does there exist a compact space $X$ such that $\mathbb{E}$ is a CW substitute for $\mathbb{F}(X)$ ? Is it true that such an $X$ exists when $\mathbb{E}$ is bounded below?

Problem 9. Characterize compact Hausdorff spaces having the same stable shape as a compact space with weight $\leq \mathfrak{m}$, where $\mathfrak{m} \geq \aleph_{0}$ is a cardinal number. Is it true that $X$ belongs to this class if and only if there is a $\mathrm{CW}$ substitute $\mathbb{W}$ for $\mathbb{E}(X)$ having no more than $\mathfrak{m}$ cells? 
Problem 10. Is it true that $\boldsymbol{\pi}$ - $\operatorname{dim} X=\operatorname{dim} X$ for every compact metric space $X$ ?

Acknowledgments. The author wishes to express his thanks to Professor Stanisław Spież for many suggestions and stimulating conversations.

\section{References}

[ $\left.\mathrm{A}_{1}\right] \quad$ J. F. Adams, Stable Homotopy and Generalized Homology, Univ. of Chicago Press, 1974.

$\left[\mathrm{A}_{2}\right] \quad-$, On the groups $J(X)-I V$, Topology 5 (1966), 21-71.

[Ba 1 F. W. Bauer, A strong shape theory admitting an S-dual, Topology Appl. 62 (1995), 207-232.

$\left[\mathrm{Ba}_{2}\right] \quad-$, A strong shape theory with S-duality, Fund. Math. 154 (1997), 37-56.

[Bo] K. Borsuk, Theory of Shape, Polish Sci. Publ., 1975.

[D-P] A. Dold and D. Puppe, Duality, trace and transfer, in: Proc. Internat. Conf. on Geometric Topology (Warszawa, 1978), PWN, 1980, 81-102.

$\left[\mathrm{D}_{1}\right] \quad$ A. N. Dranishnikov, Generalized dimension of compact metric spaces, Tsukuba J. Math. 14 (1990), 247-262

$\left[\mathrm{D}_{2}\right] \quad-$, Stable cohomotopy dimension and weakly infinite dimensional spaces, Topology Appl. 47 (1992), 79-81.

[Dy] J. Dydak, The Whitehead and the Smale theorems in shape theory, Dissertationes Math. 156 (1979).

[E-H] D. A. Edwards and H. A. Hastings, Čech and Steenrod Homotopy Theories with Applications to Geometric Topology, Springer, 1976.

[F] P. Freyd, Stable homotopy, in: Proc. Conf. Categorical Algebra (La Jolla, CA, 1965), Springer, 1966, 121-172.

[H-N] Q. Haxhibeqiri and S. Nowak, Duality between stable strong shape morphisms and stable homotopy classes, Glasnik Mat. 36 (56) (2001), 297-310.

[H] H. W. Henn, Duality in stable shape theory, Arch. Math. (Basel) 36 (1981), $327-341$.

[Hu] S. T. Hu, Homotopy Theory, Academic Press, 1959.

[K] D. S. Kahn, An example in Čech cohomology, Proc. Amer. Math. Soc. 16 (1965), 584.

[K-K-S] D. S. Kahn, J. Kaminker and C. Schochet, Generalized homology theories on compact metric spaces, Michigan Math. J. 24 (1977), 203-224.

[L] E. L. Lima, The Spanier-Whitehead duality in new homotopy categories, Summa Brasil. Math. 4 (1959), 91-148.

[Li] T. Y. Lin, Duality and Eilenberg-MacLane spectra, Proc. Amer. Math. Soc. 56 (1976), 291-299.

[L-W] A. T. Lundell and S. Weingram, The Topology of CW Complexes, Van Nostrand Reinhold, 1969.

[Ma-Se] S. Mardešić and J. Segal, Shape Theory, North-Holland, 1982.

[Ma] H. R. Margolis, Spectra and the Steenrod Algebra, North-Holland, 1983.

[Mi] T. Miyata, Generalized stable shape and duality, Topology Appl. 109 (2001), $75-88$.

[Mi-Se 1 T. Miyata and J. Segal, Generalized stable shape and the Whitehead theorem, Topology Appl. 63 (1995), 139-164. 
[Mi-Se 2 T. Miyata and J. Segal, Generalized stable shape and Brown's representation theorem, ibid. 94 (1999), 275-305.

[Mo] K. Morita, The Hurewicz and the Whitehead theorems in shape theory, Sci. Rep. Tokyo Kyoiku Daigaku Sect. A 12 (1974), 246-258.

[N] S. Nowak, On the relationships between shape properties of subcompacta of $S^{n}$ and homotopy properties of their complements, Fund. Math. 128 (1987), 47-60.

[R] T. B. Rushing, Topological Embeddings, Academic Press, New York, 1973.

$\left[\mathrm{S}_{1}\right] \quad$ E. H. Spanier, Function spaces and duality, Ann. of Math. (2) 70 (1959), 338378.

$\left[\mathrm{S}_{2}\right] \quad-$, Algebraic Topology, McGraw-Hill, New York, 1966.

$\left[\mathrm{S}_{1} \mathrm{~W}_{1}\right]$ E. H. Spanier and J. H. C. Whitehead, Duality in homotopy theory, Mathematika 2 (1955), 194-203.

$\left[\mathrm{S}-\mathrm{W}_{2}\right] \quad-,-$, Obstructions to compression, Quart. J. Math. Oxford 6 (1955), 91-100.

$\left[\mathrm{S}_{-} \mathrm{W}_{3}\right] \quad-,-$, The theory of carriers and S-theory, in: Algebraic Geometry and Topology (A Symposium in honor of S. Lefschetz), Princeton Univ. Press, 1957, $330-360$.

[Sw] R. M. Switzer, Algebraic Topology-Homotopy and Homology, Springer, 1975.

[W] C. T. C. Wall, Finiteness conditions for CW-complexes, Ann. of Math. 81 (1965), 59-69.

[Wa] T. Watanabe, On spaces which have the shape of compact metric spaces, Fund. Math. 104 (1979), 1-11.

Institute of Mathematics

University of Warsaw

Banacha 2

02-097 Warszawa, Poland

E-mail: snowak@mimuw.edu.pl

Received 19 June 2001;

in revised form 15 April 2003 and 22 December 2003 